\title{
Wake potential in graphene-insulator-graphene composite systems
}

\author{
Vito Despoja, ${ }^{1,2,3,{ }^{*}}$ Ivan Radović, ${ }^{4}$ Lazar Karbunar, ${ }^{5}$ Ana Kalinić,,${ }^{5,4}$ and Zoran L. Mišković ${ }^{6}$ \\ ${ }^{1}$ Institute of Physics, Bijenička 46, HR-10000 Zagreb, Croatia \\ ${ }^{2}$ Donostia International Physics Center (DIPC), Paseo Manuel de Lardizabal, 20018 San Sebastian, Basque Country, Spain \\ ${ }^{3}$ Universidad del Pais Vasco, Centro de Fisica de Materiales CSIC-UPV/EHU-MPC, Avenida Tolosa 72, E-20018 San Sebastian, Spain \\ ${ }^{4}$ Vinča Institute of Nuclear Sciences, University of Belgrade, PO Box 522, 11001 Belgrade, Serbia \\ ${ }^{5}$ School of Electrical Engineering, University of Belgrade, Bulevar Kralja Aleksandra 73, 11120 Belgrade, Serbia \\ ${ }^{6}$ Department of Applied Mathematics and Waterloo Institute for Nanotechnology, University of Waterloo, Waterloo, Ontario, Canada N2L $3 G 1$
}

(Received 4 June 2019; revised manuscript received 3 July 2019; published 29 July 2019)

\begin{abstract}
We study the wake potential produced by an external charged particle that moves parallel to various $\mathrm{sy}_{1}-\mathrm{Al}_{2} \mathrm{O}_{3}-\mathrm{sy}_{2}$ sandwich-like composites, where the system $\mathrm{sy}_{i}$ (with $i=1,2$ ) may be vacuum, pristine graphene, or doped graphene. The effective dielectric function of the composites is obtained using three complementary methods for graphene's electronic response, based on the massless Dirac fermions (MDF) method, the extended hydrodynamic (eHD) model, and the ab initio approach. Three velocity regimes are explored with respect to the threshold for excitations of the Dirac plasmon in graphene, given by its Fermi velocity $v_{F}$. In the low-velocity regime (below $v_{F}$ ), only the transverse optical (TO) phonons in the $\mathrm{Al}_{2} \mathrm{O}_{3}$ layer contribute to the wake potential in the surface with $\mathrm{sy}_{2}$ (which is nearest to the charged particle), in a manner that is only sensitive to the composition of that system: if $s_{2}$ is vacuum, the TO phonons give rise to intense oscillations in the wake potential, which are strongly suppressed if $s_{2}$ is pristine or doped graphene. For intermediate velocities (above $v_{F}$ ), the hybridized plasmon-TO phonon modes on both surfaces contribute to the wake potential in the surface with $\mathrm{sy}_{2}$, with the most dominant contribution coming from the hybridized Dirac-like plasmonic modes. In the high-velocity regime (well above $v_{F}$ ), the highest-lying hybridized Dirac plasmon gives the dominant contribution to the wake potential, which exhibits a typical $\mathrm{V}$-shaped wave-front pattern that lags behind the charged particle. It is found that the MDF method agrees very well with the results of the ab initio method for small and intermediate velocities. However, in the high-velocity regime, the high-energy $\pi$ plasmon in graphene introduces new features in the wake potential in the form of fast oscillations, just behind the charged particle. Those oscillations in the wake potential are well described by both the eHD and the ab initio method, proving that the $\pi$ plasmon indeed behaves as a collective mode.
\end{abstract}

DOI: 10.1103/PhysRevB.100.035443

\section{INTRODUCTION}

When a charged particle moves parallel to a metallic or polar crystal surface, it drags charges in the surface producing a dipolar density distribution (in analogy with the polaron problem), which is composed of single-particle excitations and the excitation of collective modes in the surface-plasmons or phonons, respectively. In the case of the collective modes, the excited charge density wave patterns lag behind the incident particle forming a $\mathrm{V}$ shape, known as the "wake effect" in analogy to ship waves on water. The existence of the wake pattern in a solid target is considered to be a signature of collective modes in the target material, with its parametersthe amplitude of the wake, its shape, and the spatial extentbeing controlled by the velocity of the incident particle and by its impact parameter, as well as by the dispersion relation and the damping processes of the collective modes.

Historically, the wake effect was first analyzed theoretically in 1974 by Neelavathi et al. [1], who predicted the possibility of bound electron states in the wake potential trailing a

\footnotetext{
*vito@phy.hr
}

swift ion that moves in a solid target. The first evidence of the wake effect was provided shortly afterward in an experiment demonstrating the so-called vicinage effect in the energy loss of swift ion clusters in solids [2,3]. The vicinage effect due to the plasmon wake was also predicted to give rise to strongly asymmetric Coulomb explosion patterns of fast clusters in metals and plasmas [4,5]. Moreover, the wake effect was shown to play an important role in the dynamic screening of the core exciton in the electron energy loss spectroscopy (EELS) data for semiconductors and insulators measured in a scanning transmission electron microscope (STEM) [6].

The occurrence of the wake effect was also studied on solid surfaces, both in theoretical works $[7,8]$ and in experiments $[9,10]$, which demonstrated vicinage effects in the Coulomb explosion and energy loss of diatomic molecules grazingly scattered on crystal surfaces. In the more recent experiments, it was shown that the surface wake also plays a role in the energy loss and straggling of fast ions traversing a dielectric boundary [11] and that it affects the formation of circular Rydberg states in beam-foil experiments [12]. While the above studies were concerned with the wake due to electronic collective modes in solids and their surfaces, there were several studies addressing the role of the wake due to 
the excitation of the Fuchs-Kliewer (FK) or optical surface phonon modes in ion scattering from polar surfaces $[13,14]$.

As far as the two-dimensional (2D) conductive materials are concerned, the wake effect was first studied theoretically in a strongly coupled 2D electron gas by Wang and $\mathrm{Ma}$ [15], followed by several studies of the wake effect in carbon nanostructures, including carbon nanotubes [16] and graphene [17-23]. For the latter material, the wake effect was also studied in the presence of plasmon-phonon hybridization taking place between graphene and a polar substrate [24] for both isolated [25] and correlated [26] charged particles moving parallel to graphene. There have been other investigations of the wake effect, e.g., in the case of dust grains in flowing plasma, [27] where an analogy was established with the Mach cone formation, as well as in the case of a 2D electron gas with defects, where plasmon excitation was caused by microwave radiation [28]. In addition, a recent study demonstrated efficient steering of the plasmon wakes that were excited by a free electron propagating over a nanopatterned metal surface, where the analogy with Cherenkov radiation was emphasized [29].

It should be mentioned that the scattering geometry, where an incident charged particle moves parallel to a solid surface or parallel to a $2 \mathrm{D}$ material lying atop a substrate, may be of interest, e.g., for possible experiments using grazing scattering of fast ions $[9,10]$ or for the aloof trajectories of the electron beam used for vibrational and valence EELS within STEM [30]. In analogy with the Cherenkov radiation, one expects that for the wake effect to emerge in such experiments, a threshold value should exist for the velocity of the incident particle, which needs to exceed the phase velocity of the relevant surface modes in the target material.

In a $2 \mathrm{D}$ conductive crystal, such as graphene, it is expected that a prominent role in the wake formation due to slow incident particles will be played by the so-called sheet plasmon, also known as the Dirac plasmon in doped graphene [31-34], which operates in the terahertz $(\mathrm{THz})$ to mid-infrared (midIR) frequency range. It is a unique feature of graphene that the dispersion relation of its Dirac plasmon may be efficiently tuned by changing the density of charge carriers in graphene, which can be controlled via the potential applied to external gate(s). As a consequence, one expects that the parameters of the resulting wake pattern in graphene-its amplitude, shape, and spatial extent-can be manipulated, not only by the incident particle's speed and distance from graphene but also by graphene's doping density as an externally controlled parameter. We mention that this tunability of the Dirac plasmon in graphene has already been experimentally realized, showing a great potential for applications in photonics, optoelectronics, transformation optics, and plasmonics in the $\mathrm{THz}$ to mid-IR range [35-38].

Various experimental designs of layered heterostructures involving graphene sheets include insulating spacer layers made of polar materials [39-41], which usually support strong FK phonon modes [42] that are also active in the $\mathrm{THz}$ to midIR frequency range and can therefore strongly interact with the Dirac plasmon mode(s) in the nearby graphene sheet(s). As a consequence, both the dispersion and the damping pathways of the Dirac plasmons can be altered in a substantial way $[25,26,43,44]$, affecting graphene's tunability for optoelectronic [39,42-45] and plasmonic [46-48] applications. It is expected that such strong plasmon-phonon hybridization can also affect the wake effect when the charged particle moves parallel to a heterostructure involving graphene. In that case, the wake parameters - again, its amplitude, shape, and spatial extent - can be additionally manipulated by the thickness of the polar layers or by their dielectric properties [31,49].

As a prototype of such heterostructures involving doped graphene sheets, we have studied in our previous publication [50] a sandwich-like composite graphene- $\mathrm{Al}_{2} \mathrm{O}_{3}$-graphene, and we found that it supports a variety of interesting plasmonphonon hybrid modes. Using a $5 \mathrm{~nm}$ thick layer of sapphire (aluminum oxide, $\mathrm{Al}_{2} \mathrm{O}_{3}$ ), an experimentally often used dielectric spacer $[41,48,51,52]$ which supports two prominent transverse optical (TO) phonon modes in its bulk, one obtains two strongly coupled pairs of the FK phonon modes that are localized at each surface of the sapphire layer. If one additionally assumes that two sheets of doped graphene straddle the sapphire layer, each supporting a Dirac plasmon mode, one can observe up to six well-defined hybrid modes in the $\mathrm{THz}$ to mid-IR frequency range showing a quite diverse set of dispersion relations with in-plane wave numbers $Q \lesssim 1 \mathrm{~nm}^{-1}$ [50].

In this paper we shall explore the efficiency and selectivity of the excitation of different hybrid modes in the graphene$\mathrm{Al}_{2} \mathrm{O}_{3}$-graphene composite by a charged particle that moves outside the structure and parallel to its surfaces. This will be achieved by exposing any wake patterns that may arise in the electrostatic potential in the surface closest to the incident particle. Such wake potential may be of interest for studying, e.g., the possible occurrence of the wake-riding electrons on that surface $[8,53]$. We designate our sandwich structure as $\mathrm{sy}_{1}-\mathrm{Al}_{2} \mathrm{O}_{3}-\mathrm{sy}_{2}$, where the system on either side of the sandwich, $\mathrm{sy}_{i}$ with $i=1,2$, may be vacuum, pristine graphene, denoted as gr(undoped), or doped graphene, denoted as gr(doped). In the cases of undoped and doped graphene layers, the corresponding values of the Fermi energy relative to the Dirac point will be chosen as $E_{F}=0$ and $200 \mathrm{meV}$, respectively. The surface that is closest to the incident charged particle will be assumed to be occupied by the system sy ${ }_{2}$. It can be shown that the wake potential in that surface is predominantly determined by the composition of the system $\mathrm{sy}_{2}$.

The propagator of the dynamically screened Coulomb interaction $W\left(Q, \omega, z, z^{\prime}\right)$ for a composite structure $\mathrm{sy}_{1}-\mathrm{Al}_{2} \mathrm{O}_{3}-\mathrm{sy}_{2}$ was derived in our previous paper [50], so its derivation will only be briefly outlined here. The main ingredients to our model are the single-layer graphene response functions for noninteracting electrons, $\chi_{i}^{0}(Q, \omega)$ with $i=1,2$, and the local dielectric function $\epsilon_{S}(\omega)$ of the bulk $\mathrm{Al}_{2} \mathrm{O}_{3}$. The response functions $\chi_{1,2}^{0}(Q, \omega)$ are obtained in this work at zero temperature using three complementary methods: (i) the random phase approximation (RPA) for doped graphene, taking into account just its $\pi$ electron bands in the Dirac cone approximation [54,55] with the effects of damping included via the Mermin procedure [56], which we call the massless Dirac fermions (MDF) method; (ii) a phenomenological approach that describes high-energy interband transitions in graphene by a two-fluid model for its $\pi$ and $\sigma$ electrons, amended by a low-energy Dirac contribution near the $K$ points in the Brillouin zone (BZ), which we call the extended hydrodynamic (eHD) model [57,58]; and (iii) an $a b$ initio-RPA method that includes 
transitions between all valence and conduction bands in graphene, which we simply call the $a b$ initio method. The propagator $W$ is used to derive an effective $2 \mathrm{D}$ dielectric function, $\epsilon(Q, \omega)$, of the composite $\mathrm{sy}_{1}-\mathrm{Al}_{2} \mathrm{O}_{3}-\mathrm{sy}_{2}$ structure, which is further used to evaluate the wake potential and to facilitate understanding of the appearance and the role of certain modes by analyzing the peaks in the loss function of the structure, given by $\operatorname{Im}[-1 / \epsilon(Q, \omega)][56,59]$.

The wake effect is explored in three regimes of the incident particle velocity, $v$, which will be expressed in units of the Fermi velocity of graphene's $\pi$ electron bands in the Dirac cone approximation [32], having a value of $\approx c / 300$ (where $c$ is the speed of light in vacuum). It was found in Ref. [17] that the threshold velocity for observing the wake effect in graphene due to the excitation of its Dirac plasmon is given by its Fermi velocity, i.e., $v_{\mathrm{thr}}=v_{F}=1$ in our units. Accordingly, in the low-velocity regime, we set $v=0.5$, so that only the phonon-like modes can contribute to the wake potential in the surface closest to the incident particle. It will be then shown that, if the system $\mathrm{sy}_{2}$ is vacuum, the TO phonons give rise to strong oscillations in the wake potential, which are strongly suppressed if $\mathrm{sy}_{2}$ is either gr(doped) or gr(undoped) because graphene screens out the TO phonons in both cases. For the intermediate velocity of $v=2$, which exceeds the threshold velocity for the Dirac plasmon excitation, the incident particle excites hybridized TO phonon-Dirac plasmon modes, giving rise to intense features in the wake potential. When $\mathrm{sy}_{2}$ is vacuum, the TO phonons still give rise to strong oscillations in the wake potential, which are reduced when sy is $\operatorname{gr}\left(\right.$ doped) and almost disappear when $\mathrm{sy}_{2}$ is gr(undoped). In the high-velocity regime, where we set $v=4$, strong Dirac plasmon-like modes become the dominant contribution to the wake potential, which behaves as a perfect (single-harmonic), $\mathrm{V}$-shaped wave lagging behind the charged particle.

It will be shown that the MDF method agrees very well with the $a b$ initio method for small and intermediate velocities. However, in the high-velocity limit, smaller wave numbers become important for the wake effect, where the so-called $\pi$ plasmon in graphene exhibits a strongly dipolar mode [60], which introduces new features (fast oscillations) in the wake potential. It is shown that, in this regime, the eHD model agrees very well with the $a b$ initio method. We note that while all three methods provide results for $\chi_{i}^{0}(Q, \omega)$ including a full dependence on the wave number $Q$, we shall see that the so-called optical limit of each method, when $Q \approx 0$, can play an especially useful role in our calculations.

In Sec. II we outline the methodology used to calculate dynamically screened Coulomb interaction $W\left(Q, \omega, z, z^{\prime}\right)$, the effective $2 \mathrm{D}$ dielectric function $\epsilon(Q, \omega)$, and the wake potential in various $\mathrm{sy}_{1}-\mathrm{Al}_{2} \mathrm{O}_{3}-\mathrm{sy}_{2}$ structures. In Sec. III we present and discuss our results, whereas in Sec. IV we give our concluding remarks.

\section{METHODOLOGY}

\section{A. Modeling of the system}

We assume that a point-like particle with charge Ze moves at the velocity $v$ parallel to a graphene-insulator-graphene composite at distance $z_{0}$ from the closest surface, as schemat-

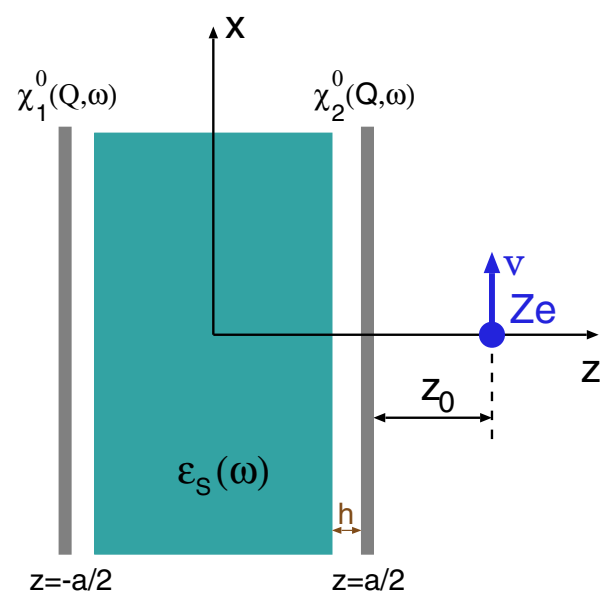

FIG. 1 . The charged particle $(\mathrm{Ze})$ moves with velocity $v$ parallel to the gr- $\mathrm{Al}_{2} \mathrm{O}_{3}$-gr composite. Simplified model where the sapphire layer is approximated by a homogeneous dielectric slab described by local dielectric function $\epsilon_{S}(\omega)$, whereas graphene sheets are described by $2 \mathrm{D}$ response functions for noninteracting electrons, $\chi_{1,2}^{0}(\mathbf{Q}, \omega)$.

ically shown in Fig. 1. Two graphene layers are allowed to occupy the $z=\mp a / 2$ planes, with $h$ being the size of the air gaps to the nearby insulator surfaces.

The surface polarization waves excited by the moving charge can be studied in terms of the total potential at the $z=a / 2$ plane, which may be expressed as $[7,8,18,25,61]$

$$
\begin{aligned}
\Phi^{\mathrm{tot}}(\rho, z=a / 2, t)= & \frac{2 Z e}{\pi} \int_{0}^{\infty} d Q_{x} \int_{0}^{\infty} d Q_{y} \cos \left(Q_{y} y\right) \\
& \times \frac{e^{-Q_{0}}}{Q} \operatorname{Re}\left\{\frac{e^{i Q_{x}(x-v t)}}{\epsilon\left(\mathbf{Q}, \omega=Q_{x} v\right)}\right\},
\end{aligned}
$$

which we shall call the wake potential. Here $\mathbf{Q}=\left(Q_{x}, Q_{y}\right)$ is the momentum transfer vector parallel to the $(x, y)$ plane, $Q=\sqrt{Q_{x}^{2}+Q_{y}^{2}}$, and $\rho=(x, y)$ is a $2 \mathrm{D}$ position vector in that plane. The main quantity in (1) is the effective $2 \mathrm{D}$ dielectric function $\epsilon(\mathbf{Q}, \omega)$, which may be physically interpreted as the dielectric response squeezed in the $z=a / 2$ plane. We note that with the incident particle moving parallel to the $x$ axis, the kinematic resonance condition is accounted for by setting $\omega=Q_{x} v$ in the expression for $\epsilon(\mathbf{Q}, \omega)$ in Eq. (1).

On the other hand, it can be shown [56] that the probability density for the parallel momentum transfer $\mathbf{Q}$ and the energy loss $\omega$ in the reflection EELS (REELS) is proportional to the imaginary part of the induced potential $W^{\text {ind }}=\frac{2 \pi}{Q}[1 / \epsilon-1]$, i.e.,

$$
P(\mathbf{Q}, \omega) \propto-\operatorname{Im} \frac{1}{\epsilon(\mathbf{Q}, \omega)},
$$

with the quantity on the right-hand side called the energy loss function.

A derivation of the effective 2D dielectric function is described in detail in Ref. [50], so we only briefly review the main steps here. From Eqs. (16), (18), (23), and (24) of Ref. [50] it can be shown that the Fourier transform of the screened Coulomb interaction in the plane in which 
$z=z^{\prime}=a / 2$ can be written as

$$
W\left(\mathbf{Q}, \omega, z=z^{\prime}=a / 2\right)=\frac{\left(\tilde{v}_{11}+\tilde{v}_{12}^{2} \tilde{\chi}_{1}\right)\left(1+\tilde{v}_{11} \tilde{\chi}_{2}\right)}{1-\tilde{v}_{12}^{2} \tilde{\chi}_{1} \tilde{\chi}_{2}},
$$

where the RPA screened response functions of the two graphene layers are

$$
\tilde{\chi}_{i}(\mathbf{Q}, \omega)=\frac{\chi_{i}^{0}(\mathbf{Q}, \omega)}{1-\tilde{v}_{11}(Q) \chi_{i}^{0}(\mathbf{Q}, \omega)}, \quad i=1,2,
$$

whereas the "intrasystem" and "intersystem" Coulomb interactions, "screened" by the dielectric slab, have the following analytical form:

$$
\begin{gathered}
\tilde{v}_{11}=v_{Q}\left\{1+D_{S}(\omega) \frac{1-e^{-2 Q a}}{1-D_{S}^{2}(\omega) e^{-2 Q a}}\right\}, \\
\tilde{v}_{12}=v_{Q} \frac{1-D_{S}^{2}(\omega)}{1-D_{S}^{2}(\omega) e^{-2 Q a}} e^{-Q a}
\end{gathered}
$$

with

$$
D_{S}(\omega)=\frac{1-\epsilon_{S}(\omega)}{1+\epsilon_{S}(\omega)}
$$

and $v_{Q}=\frac{2 \pi}{Q}$. Since we are only interested in the polarization of the structure in the plane $z=a / 2$, the effective 2D dielectric function may be defined as the ratio of the total potential in that plane to the bare in-plane Coulomb interaction, viz.,

$$
\epsilon^{-1}(\mathbf{Q}, \omega)=W\left(\mathbf{Q}, \omega, z=z^{\prime}=a / 2\right) / v_{Q} .
$$

After inserting Eqs. (3)-(6) into Eq. (7) and doing some algebra, the effective $2 \mathrm{D}$ dielectric function may be written in a more explicit form as

$$
\begin{aligned}
\epsilon(\mathbf{Q}, \omega)= & \frac{1}{2}\left[1+\epsilon_{S} \operatorname{coth}(Q a)-2 v_{Q} \chi_{2}^{0}\right] \\
& -\frac{1}{2} \frac{\epsilon_{S}^{2} \operatorname{cosech}^{2}(Q a)}{1+\epsilon_{S} \operatorname{coth}(Q a)-2 v_{Q} \chi_{1}^{0}},
\end{aligned}
$$

in terms of the alumina local dielectric function $\epsilon_{S}(\omega)$ and the $2 \mathrm{D}$ response functions $\chi_{1,2}^{0}(\mathbf{Q}, \omega)$ of noninteracting electrons in the graphene layers. The latter are calculated using three methods: MDF, eHD, and $a b$ initio method.

\section{B. Graphene response functions $\chi_{1,2}^{0}$ : MDF method}

Analytical expressions for the response function $\chi_{i}^{0}(Q, \omega)$ of single-layer doped graphene were obtained at zero temperature in Refs. [54,55] within the collisionless RPA for graphene's $\pi$ electron bands treated in the Dirac cone approximation. A procedure was further outlined in Refs. [35,56] that includes the effects of electron collisions in $\chi_{i}^{0}(Q, \omega)$ following Mermin's prescription [62], giving rise to the MDF method used in our work. This method accurately describes both the interband and the intraband electron transition at frequencies up to the IR for typical doping densities $n$ of graphene. In particular, there are three types of electronic excitations that occur in different regions in the first quadrant of the $(Q, \omega)$ plane [54,55]. A continuum of the interband electron-hole excitations occurs in the region $\omega / v_{F}>\max \left(Q, 2 k_{F}-Q\right)$, while the continuum of the intraband electron-hole excitations occurs in the region $Q>$ $\omega / v_{F}>\max \left(Q-2 k_{F}, 0\right)$, where $k_{F}=\sqrt{\pi|n|}$ is graphene's Fermi wave number. In the case of intrinsic graphene $(n=0)$, the response function only gives rise to the continuum of interband electron-hole excitations for $\omega>Q v_{F}$. The Dirac plasmon mode only arises in the case of doped graphene with a well-defined $\omega \propto \sqrt{q}$ dispersion relation traversing the region $Q<\omega / v_{F}<2 k_{F}-Q$ for $Q<k_{F}$. We note that any collective modes with dispersion relations that are immersed into the continua of electron-hole excitations arising from the MDF method are expected to undergo strong Landau damping.

\section{Optical limit}

A very simple approximation for the response function $\chi_{i}^{0}(Q, \omega)$ may be obtained within the MDF method, giving a very popular and versatile approximation for photonic applications of graphene in the THz to mid-IR range of frequencies. This approximation rests on the so-called optical limit for 2D conductive materials, where $Q \approx 0$, so that the independentelectron response function is expressed in terms of the optical conductivity of the material, $\sigma_{i}(\omega)$, as [63]

$$
\chi_{i}^{0}(Q, \omega)=\frac{Q^{2}}{i \omega} \sigma_{i}(\omega)
$$

The optical conductivity in doped graphene may be generally written in terms of the intraband and interband contributions as [34]

$$
\sigma_{i}(\omega)=\sigma_{i}^{\text {intra }}(\omega)+\sigma_{i}^{\text {inter }}(\omega), \quad i=1,2 .
$$

The intraband term in this expression only arises when graphene is doped, and it has a generic Drude form, which may be written at zero damping as $\sigma_{i}^{\text {intra }}(\omega)=i \frac{e^{2} E_{F}}{\pi \hbar^{2} \omega}$, whereas the real part of the interband term is given by $\operatorname{Re}\left[\sigma_{i}^{\text {inter }}(\omega)\right]=$ $\sigma_{0} \Theta\left(\omega-2 E_{F} / \hbar\right)$, with its imaginary part determined by the Kramers-Kronig relation. Here, $\Theta$ is the unit step function expressing the Pauli blocking in doped graphene, whereas $\sigma_{0} \equiv e^{2} /(4 \hbar) \approx c / 548$ is the universal conductivity of intrinsic graphene $\left(E_{F}=0\right)$, resulting from the low-energy $\pi \rightarrow$ $\pi^{*}$ electron transitions near the $K$ points of the BZ [32], where the Dirac cone approximation applies. Using these simple expressions for the intraband and interband conductivities in Eq. (10), we obtain a response function from Eq. (9), which we call the "optical" MDF method.

\section{Graphene response functions $\chi_{1,2}^{0}$ : The eHD method}

At higher frequencies, corresponding to $1 \mathrm{eV} \lesssim \hbar \omega \lesssim$ $50 \mathrm{eV}$, which are of interest in the valence EELS, the effects of doping are not important, but the onset of high-energy interband electron transitions in graphene gives rise to two prominent peaks in the energy loss spectra near $4 \mathrm{eV}$ and $14 \mathrm{eV}$, known as the $\pi$ and $\sigma+\pi$ plasmon peaks [64], respectively. It was found that several different sets of experimental EELS data on single-layer [58] and multilayer [65] graphene can be successfully modeled by resorting to the optical limit for the response function $\chi_{i}^{0}(Q, \omega)$, given in Eq. (9). Moreover, it was found that it suffices to only consider the conductivity of intrinsic graphene $\left(E_{F}=0\right)$ when modeling the main peaks in the EELS data $[58,64,65]$, so 
we may use Eq. (10) with $\sigma_{i}(\omega)=\sigma_{i}^{\text {inter }}(\omega)$, describing both $\pi \rightarrow \pi^{*}$ and $\sigma \rightarrow \sigma^{*}$ interband electron transitions across the BZ in graphene.

A simple empirical approximation for the interband conductivity, $\sigma_{i}^{\text {inter }}(\omega) \approx \sigma_{\mathrm{HD}}(\omega)$, was obtained based on a $2 \mathrm{D}$ planar version of the two-fluid hydrodynamic (HD) model for graphene's $\pi$ and $\sigma$ electrons [57], which consists of two terms, $\sigma_{\mathrm{HD}}(\omega)=\sigma_{\pi}(\omega)+\sigma_{\sigma}(\omega)$. Here, the optical conductivity of the $\nu$ th fluid (with $v=\pi, \sigma$ ) was obtained in a Drude-Lorentz form,

$$
\sigma_{v}(\omega)=i e^{2} \frac{n_{v}^{0}}{m_{v}^{*}} \frac{\omega}{\omega\left(\omega+i \beta_{v}\right)-\omega_{v r}^{2}},
$$

with $n_{v}^{0}, m_{v}^{*}, \omega_{v r}$, and $\beta_{v}$ being the equilibrium number density of electrons per unit area, effective electron mass, restoring frequency, and the broadening factor for the $v$ th electron fluid, respectively.

Noting that the restoring frequency of $\omega_{\pi r} \sim 4 \mathrm{eV}$ in the $\sigma_{\pi}(\omega)$ term of the HD model describes electron transitions between the $\pi$ and $\pi^{*}$ bands at the $M$ points in the BZ of graphene, it turns out that $\sigma_{\mathrm{HD}}(\omega)$ may be used as a very good approximation for modeling the EELS data for graphene at frequencies $\omega \gtrsim 2 \mathrm{eV}$ [59]. However, this model gives $\operatorname{Re}\left[\sigma_{\mathrm{HD}}(\omega \rightarrow 0)\right]=0$, in contrast to the well-established result for the zero-frequency limit of the interband conductivity in intrinsic graphene, $\operatorname{Re}\left[\sigma_{i}^{\text {inter }}(\omega \rightarrow 0)\right]=\sigma_{0}$.

At the same time, there were indications in recent experimental EELS data $[58,64,66,67]$ that the Dirac physics of the low-energy interband transitions plays an important role at the energy losses $\lesssim 2 \mathrm{eV}$ for intrinsic graphene. Accordingly, we have extended the above HD model by including a low-energy contribution describing the $\pi \rightarrow \pi^{*}$ transitions near the $K$ points in the BZ, which we call the Dirac correction, $\sigma_{D}(\omega)$, having the proper zero-frequency limit $\operatorname{Re}\left[\sigma_{D}(\omega \rightarrow 0)\right]=\sigma_{0}$. As a result, we obtained the eHD model as [58]

$$
\sigma_{\mathrm{eHD}}(\omega)=\sigma_{D}(\omega)+f \sigma_{\pi}(\omega)+\sigma_{\sigma}(\omega),
$$

where

$$
\sigma_{D}(\omega)=\sigma_{0} \frac{\omega_{c}^{4}}{\omega_{c}^{4}+\omega^{4}}\left[1+i \frac{\sqrt{2}}{2} \frac{\omega}{\omega_{c}}\left(1+\frac{\omega^{2}}{\omega_{c}^{2}}\right)\right] .
$$

Here, $\omega_{c}$ is a suitable cutoff frequency for the Dirac contribution, whereas the parameter $f \in[0,1]$ in Eq. (12) describes a reduction of the contribution from the $\pi \rightarrow \pi^{*}$ transitions at the $M$ points of the BZ. By taking $m_{v}^{*}$ in Eq. (11) to be the free electron mass $m_{e}$ for both the $\pi$ and $\sigma$ electron fluids, one can use the $f$-sum rule for electron transitions in graphene to express $f$ in terms of $\omega_{c}$ as [58]

$$
f=1-\frac{\sqrt{2}}{8} \frac{m_{e} \omega_{c}}{\hbar n_{a t}},
$$

where $n_{a t}$ is the atomic density of graphene. It should be noted that this value of $f$ ensures that the first two terms on the righthand side of Eq. (12) fulfill the $f$-sum rule for one $\pi$ electron per carbon atom in graphene.

\section{Graphene response functions $\chi_{1,2}^{0}: A b$ initio method}

If the graphene layers are considered to be true $2 \mathrm{D}$ systems, their full nonlocal independent-electron response functions may be written as [33]

$$
\mathcal{X}_{i}^{0}\left(\mathbf{Q}, \omega, z, z^{\prime}\right)=\chi_{i}^{0}(\mathbf{Q}, \omega) \delta\left(z-z_{i}\right) \delta\left(z^{\prime}-z_{i}\right), \quad i=1,2,
$$

where $z_{1}=-a / 2$ and $z_{2}=a / 2$. Here, the $\chi_{1,2}^{0}(\mathbf{Q}, \omega)$ functions are calculated from first principles as

$$
\chi_{i}^{0}(\mathbf{Q}, \omega)=L \chi_{\mathbf{G}=0 \mathbf{G}^{\prime}=0}^{0, i}(\mathbf{Q}, \omega), \quad i=1,2,
$$

where the 3D Fourier transform of the independent-electron response function is given by

$$
\begin{aligned}
& \chi_{\mathbf{G}^{\prime}}^{0, i}(\mathbf{Q}, \omega)= \frac{2}{\Omega} \sum_{\mathbf{K} \in S B Z} \sum_{n, m} \frac{f_{n}^{i}(\mathbf{K})-f_{m}^{i}(\mathbf{K}+\mathbf{Q})}{\hbar \omega+i \eta+E_{n}(\mathbf{K})-E_{m}(\mathbf{K}+\mathbf{Q})} \\
& \times \rho_{n \mathbf{K}, m \mathbf{K}+\mathbf{Q}}(\mathbf{G}) \rho_{n \mathbf{K}, m \mathbf{K}+\mathbf{Q}^{*}\left(\mathbf{G}^{\prime}\right),} \\
& i=1,2,
\end{aligned}
$$

with $f_{n}^{1,2}(\mathbf{K})=\left[e^{\left(E_{n \mathbf{K}}-E_{F}^{1,2}\right) / k T}+1\right]^{-1}$ being the Fermi-Dirac distributions at the temperature $T$. The charge vertices in (17) have the form

$$
\rho_{n \mathbf{K}, m \mathbf{K}+\mathbf{Q}}(\mathbf{G})=\int_{\Omega} d \mathbf{r} e^{-i(\mathbf{Q}+\mathbf{G}) \cdot \mathbf{r}} \phi_{n \mathbf{K}}^{*}(\mathbf{r}) \phi_{n \mathbf{K}+\mathbf{Q}}(\mathbf{r}),
$$

where $\mathbf{G}=\left(\mathbf{G}_{\|}, G_{z}\right)$ are the $3 \mathrm{D}$ reciprocal lattice vectors and $\mathbf{r}=(\rho, z)$ is a $3 \mathrm{D}$ position vector. Integration in (18) is performed over the normalization volume $\Omega=S \times L$, where $S$ is the normalization surface and $L$ is a superlattice constant in the $z$ direction (separation between graphene layers in a superlattice arrangement). The plane-wave expansion of the wave function has the form

$$
\phi_{n \mathbf{K}}(\rho, z)=\frac{1}{\sqrt{\Omega}} e^{i \mathbf{K} \cdot \rho} \sum_{\mathbf{G}} C_{n \mathbf{K}}(\mathbf{G}) e^{i \mathbf{G} \cdot \mathbf{r}},
$$

where the coefficients $C_{n \mathbf{K}}$ are obtained by solving the local density approximation-Kohn-Sham (LDA-KS) equations self-consistently, as will be discussed in Sec. III A.

\section{Optical limit}

The above $a b$ initio calculation of graphene response functions $\chi_{1,2}^{0}(\mathbf{Q}, \omega)$ is straightforward, but it is not sufficient if we want to investigate hybridization between the Dirac plasmon and the FK phonons in a dielectric surface. Namely, due to very low energy of the FK phonons ( $\sim 50 \mathrm{meV})$, the crossing with the Dirac plasmon dispersion relation occurs for very small wave numbers, on the order of $Q=0.01 \mathrm{~nm}^{-1}$, as will be demonstrated in Sec. IIIB. On the other hand, even for very dense $K$-point mesh sampling, such as $601 \times 601 \times 1$ used in this calculation, the minimum transfer wave number $Q$ that can be reached (e.g., $Q=0.049 \mathrm{~nm}^{-1}$ in this calculation) is still larger than the FK phonon-Dirac plasmon crossing wave number. Therefore, we have to find a way to calculate $\chi_{1,2}^{0}(\mathbf{Q}, \omega)$ for a finer $Q$-point mesh, allowing us to approach the optical limit when $Q \approx 0$. One possibility is that, instead of calculating the full response functions $\chi_{i}^{0}(\mathbf{Q}, \omega)$, we use the approximation given in Eq. (9) and calculate the optical conductivities $\sigma_{i}(\omega)$, which can be obtained in the strict $Q=0$ limit with good accuracy.

The optical conductivity in graphene may be written as in Eq. (10) [34], with the intraband, or Drude conductivity, 
given by

$$
\sigma_{i}^{\text {intra }}(\omega)=\frac{i n_{i}}{\omega+i \eta_{\text {intra }}}
$$

where

$$
n_{i}=-\frac{2}{\Omega} \sum_{\mathbf{K}, n} \frac{\partial f_{n}^{i}(\mathbf{K})}{\partial E_{n}(\mathbf{K})}\left|j_{n \mathbf{K}, n \mathbf{K}}^{x}(\mathbf{G}=0)\right|^{2}
$$

represents the effective number of charge carriers. The interband conductivity is

$$
\begin{aligned}
\sigma_{i}^{\text {inter }}(\omega)= & \frac{-2 i}{\omega \Omega} \sum_{\mathbf{K}, n \neq m} \frac{\hbar \omega}{E_{n}(\mathbf{K})-E_{m}(\mathbf{K})} \\
& \times \frac{f_{n}^{i}(\mathbf{K})-f_{m}^{i}(\mathbf{K})}{\hbar \omega+i \eta_{\text {inter }}+E_{n}(\mathbf{K})-E_{m}(\mathbf{K})} \\
& \times j_{n \mathbf{K}, m \mathbf{K}}^{x}(\mathbf{G}=0)\left[j_{n \mathbf{K}, m \mathbf{K}}^{x}\left(\mathbf{G}^{\prime}=0\right)\right]^{*},
\end{aligned}
$$

where the current vertices are given by

$$
j_{n \mathbf{K}, m \mathbf{K}+\mathbf{Q}}^{\mu}(\mathbf{G})=\int_{\Omega} d \mathbf{r} e^{-i(\mathbf{Q}+\mathbf{G}) \cdot \mathbf{r}} j_{n \mathbf{K}, m \mathbf{K}+\mathbf{Q}}^{\mu}(\mathbf{r}),
$$

with

$$
\begin{aligned}
& j_{n \mathbf{K}, m \mathbf{K}+\mathbf{Q}}^{\mu}(\mathbf{r}) \\
& \quad=\frac{\hbar e}{2 i m}\left\{\phi_{n \mathbf{K}}^{*}(\mathbf{r}) \partial_{\mu} \phi_{m \mathbf{K}+\mathbf{Q}}(\mathbf{r})-\left[\partial_{\mu} \phi_{n \mathbf{K}}^{*}(\mathbf{r})\right] \phi_{m \mathbf{K}+\mathbf{Q}}(\mathbf{r})\right\} .
\end{aligned}
$$

We note that in Ref. [58], we have gauged our parametrization of the empirical $\sigma_{\mathrm{eHD}}(\omega)$ using the results for $\sigma_{i}^{\text {inter }}(\omega)$ obtained from $a b$ initio calculations for undoped graphene based on Eq. (21) in a full range of frequencies.

In the optical limit, i.e., when $Q \approx 0$, one can use Eq. (9) to express the independent-electron response functions in terms of the $a b$ initio optical conductivity via Eq. (10). This approximation for $\chi_{i}^{0}(Q, \omega)$ will be called the "optical" $a b$ initio response function of doped graphene. Needless to say, this approximation should work extremely well in the technologically interesting range of frequencies from the mid-IR $\left(Q \sim 0.001 \mathrm{~nm}^{-1}\right)$ down to $\mathrm{THz}\left(Q \sim 10^{-5} \mathrm{~nm}^{-1}\right)$. Therefore, the calculation of the loss function in Eq. (2) and the integration in the wake potential in Eq. (1) in the range of wave numbers $Q<0.01 \mathrm{~nm}^{-1}$ pertaining to the optical limit will be performed using the $2 \mathrm{D}$ dielectric function given in Eq. (8) with the explicit use of the optical ab initio response function.

\section{E. $\mathrm{Al}_{2} \mathrm{O}_{3}$ model dielectric function}

Within the model used here, the $\mathrm{Al}_{2} \mathrm{O}_{3}$ slab is treated as a homogeneous dielectric described by some local dielectric function $\epsilon_{S}(\omega)$. For simplicity, we also take $h=0$, so that the dielectric layer completely fills the space between graphene layers and has the thickness of $a=5 \mathrm{~nm}$. It was found that this approximation has negligible effects on the coupled modes and therefore on the wake potential. The dielectric properties of the bulk ionic crystals in the long-wavelength limit are governed by their optical phonons in the $\Gamma$ point. Complex polar crystals, such as $\mathrm{Al}_{2} \mathrm{O}_{3}$, possess a number of different optical phonons with different symmetries and polarizations. However, here we assume that the polarization of the bulk $\mathrm{Al}_{2} \mathrm{O}_{3}$ mainly comes from excitations of the two optical modes that have the largest oscillator strengths [42,68], so that the bulk dielectric function of this material may be approximated by [69]

$$
\begin{aligned}
\epsilon_{S}(\omega)= & \epsilon_{o x}^{\infty}+\left(\epsilon_{o x}^{i}-\epsilon_{o x}^{\infty}\right) \frac{\omega_{\mathrm{TO} 2}^{2}}{\omega_{\mathrm{TO} 2}^{2}-\omega^{2}-i \omega \gamma_{\mathrm{TO} 2}} \\
& +\left(\epsilon_{o x}^{0}-\epsilon_{o x}^{i}\right) \frac{\omega_{\mathrm{TO} 1}^{2}}{\omega_{\mathrm{TO} 1}^{2}-\omega^{2}-i \omega \gamma_{\mathrm{TO} 1}},
\end{aligned}
$$

where $\epsilon_{o x}^{\infty}, \epsilon_{o x}^{i}$, and $\epsilon_{o x}^{0}$ are the optical, intermediate, and static permittivities, $\omega_{\mathrm{TO} 1}$ and $\omega_{\mathrm{TO} 2}$ are the first and second transverse optical (TO) angular frequencies (with $\omega_{\mathrm{TO} 1}<\omega_{\mathrm{TO} 2}$ ), and $\gamma_{\mathrm{TO} 1}$ and $\gamma_{\mathrm{TO} 2}$ are the damping rates of the corresponding TO phonons.

\section{RESULTS AND DISCUSSION}

\section{A. Computational details}

The first part of our $a b$ initio calculations consists of determining the KS ground state of single-layer graphene and the corresponding wave functions $\phi_{n \mathbf{K}}(\rho, z)$ and energies $E_{n}(\mathbf{K})$. For graphene's unit-cell constant we use the experimental value of $a_{g}=0.245 \mathrm{~nm}$ [70], while for the superlattice unit-cell constant (separation between the periodic replicas of graphene layers) we take $L=5 a_{g}$. For calculating the $\mathrm{KS}$ wave functions and energies we use a plane-wave, selfconsistent field DFT code (PWSCF) within the QUANTUM ESPRESSO (QE) package [71]. The core-electron interaction is approximated by the norm-conserving pseudopotentials [72], and the exchange correlation (XC) potential by the Perdew-Zunger local density approximation (LDA) [73]. To calculate the ground-state electronic density we use a $21 \times$ $21 \times 1$ Monkhorst-Pack $K$-point mesh [74] of the first BZ For norm-conserving pseudopotentials and alkaline, alkalineearth, and lighter atoms in the III, IV, V, and VI columns of the periodic table $(\mathrm{Al}, \mathrm{C}, \mathrm{S}, \mathrm{P}, \mathrm{O}, \mathrm{B}, \ldots)$ the plane-wave cutoff energy of $50 \mathrm{Ry}$ is a well-converged value for the ground-state band structure $E_{n \mathbf{K}}$ and KS wave function $\phi_{n \mathbf{K}}^{*}(\mathbf{r})$ calculations [33,75-77]. For charge vertices (18) and then response function (17) calculations the plane-wave cutoff energy can be even reduced, in comparison with that used in the ground-state calculation, and the dielectric response calculation will still be well converged [33]. Therefore the 50 Ry plane-wave cutoff energy used here gives accurate KS wave functions $\phi_{n \mathbf{K}}^{*}(\mathbf{r})$ and therefore response function $\chi^{0}$. The second part of our ab initio calculations consists of determining the independent-electron response function, Eq. (17), and the optical conductivity, Eqs. (10), (19), and (21). In order to achieve better resolution in the long-wavelength limit $(Q \approx$ $0)$ and the low-energy $(\omega \approx 0)$ limit, the response function in Eqs. (17) and (18) and the conductivity in Eqs. (10) and (19)-(23) are evaluated from the wave functions $\phi_{n \mathbf{K}}(\mathbf{r})$ and energies $E_{n}(\mathbf{K})$ calculated for the $601 \times 601 \times 1$ MonkhorstPack $K$-point mesh, which corresponds to $361801 K$ points in the first Brillouin zone (1BZ). The band summations $(n, m)$ in Eqs. (17), (20), and (21) are performed over 30 bands. In the calculations, we use two kinds of damping parameters: $\eta_{\text {intra }}=10 \mathrm{meV}$ for transitions within the same bands 
$(n \leftrightarrow n)$, and $\eta_{\text {inter }}=50 \mathrm{meV}$ for transitions between different bands $(n \leftrightarrow m)$.

For our empirical eHD method, which relies on the optical conductivity given in Eq. (12) along with Eqs. (11), (13), and (14), we have found in Ref. [58] that the parameters $n_{\pi}^{0}=n_{a t}=38 \mathrm{~nm}^{-2}, n_{\sigma}^{0}=3 n_{a t}=114 \mathrm{~nm}^{-2}, \omega_{\pi r}=$ $4.19 \mathrm{eV}, \omega_{\sigma r}=14.15 \mathrm{eV}, \beta_{\pi}=2.04 \mathrm{eV}, \beta_{\sigma}=2.178 \mathrm{eV}$, and $\omega_{c}=3.54 \mathrm{eV}$ give $\sigma_{\mathrm{eHD}}(\omega)$, which reproduces several sets of the experimental EELS data for undoped graphene and agrees well with the $a b$ initio calculations of $\sigma_{i}^{\text {inter }}(\omega)$ based on Eq. (21).

For the bulk $\mathrm{Al}_{2} \mathrm{O}_{3}$ dielectric function given in Eq. (24), we use the parameters $\epsilon_{o x}^{0}=12.53, \epsilon_{o x}^{i}=7.27, \epsilon_{o x}^{\infty}=3.20$, $\omega_{\mathrm{TO} 1}=48.18 \mathrm{meV}, \quad \omega_{\mathrm{TO} 2}=71.41 \mathrm{meV}, \quad \gamma_{\mathrm{TO} 1}=1.74 \mathrm{meV}$, and $\gamma_{\mathrm{TO} 2}=6.82 \mathrm{meV}$. After determining the response function in Eq. (17) [or (9) in the optical $Q \approx 0$ limit] and using the $\mathrm{Al}_{2} \mathrm{O}_{3}$ dielectric function in Eq. (24), the effective $2 \mathrm{D}$ dielectric function in Eq. (8) is calculated.

While the $a b$ initio method is expected to describe electronic excitations in graphene in a broad range of frequencies, up to the far ultraviolet (FUV), the MDF method provides a very good description of the electronic excitations in the $\mathrm{THz}$ to IR frequency range. The main contribution to the wake effect at low and intermediate velocities of the incident particle comes from the hybridized Dirac plasmon-TO phonon modes, which lie exactly in the $\mathrm{THz}$ to IR frequency range, so that the MDF method should be sufficient to provide a satisfactory description of the wake potential. However, for higher velocities, the high-energy $\pi$ plasmon peak (near $4 \mathrm{eV}$ ) in the energy loss spectra of graphene starts to contribute in the wake potential, and in order to capture this contribution, the MDF should be replaced by the eHD or the $a b$ initio method.

\section{B. Loss function}

In order to understand contributions of particular collective modes to the wake potential, we shall first briefly analyze the loss function intensities in several of the most relevant $\mathrm{sy}_{1}-\mathrm{Al}_{2} \mathrm{O}_{3}-\mathrm{sy}_{2}$ composites. A very detailed analysis of the loss function in various related composites can be found in Ref. [50]. The loss function intensities are calculated using the MDF method, which was found to give a sufficiently accurate wake potential for low and intermediate velocities of the incident particle. For higher velocities, the eHD or the $a b$ initio-RPA method will have to be used in order to give a more realistic description of the wake potential.

Figure 2(a) shows the loss function in the symmetric gr(doped)-gr(doped) composite, with vacuum between the graphene layers separated by $a=5 \mathrm{~nm}$. The thin white lines show the lower, $\omega=v_{F}\left(Q-2 k_{F}\right)$, and the upper, $\omega=v_{F} Q$, edges of the intraband $\pi^{*} \leftrightarrow \pi^{*}$ electron-hole excitation continuum, as well as the lower edge, $\omega=2 E_{F}-v_{F} Q$, of the interband $\pi \leftrightarrow \pi^{*}$ electron-hole excitation continuum in the Dirac cone approximation. We see two plasmon branches, which are a consequence of hybridization between the Dirac plasmons in graphene layers. Because the interaction which produces plasmon hybridization decays as $\sim e^{-Q a}$, a "cutoff" wave number below which two plasmons still interact may be estimated as $Q_{C} \sim 1 / a$. Therefore, for $Q>Q_{C}$ the plasmons interact weakly, and the two branches become almost degenerate. The loss function intensity shows that this estimate works quite well, as the Dirac plasmon splitting occurs below $Q_{C} \sim 0.2 \mathrm{~nm}^{-1}$, while above that value the two plasmons tend to merge. Considering that the system is symmetric, the parity is a good quantum number and the plasmon modes can be classified as symmetric (or even) and antisymmetric (or odd) modes. The dotted magenta and white lines in Fig. 2(a) show the dispersion relations of the odd $\left(\omega_{-}\right)$and even $\left(\omega_{+}\right)$Dirac plasmons, calculated analytically using the "optical" MDF model [50]. The agreement with the full MDF model loss function intensity patterns worsens for larger wave numbers $\left(Q \gtrsim 0.2 \mathrm{~nm}^{-1}\right)$, as excepted.

Let us now examine what happens when a dielectric slab is placed between two graphene layers. Figure 2(b) shows the loss function in the symmetric gr(doped)- $\mathrm{Al}_{2} \mathrm{O}_{3}$-gr(doped) composite. Considering that now each doped graphene supports one Dirac plasmon and each $\mathrm{Al}_{2} \mathrm{O}_{3}$ surface supports two FK phonons, this system supports six hybridized modes in total. The dotted lines show the dispersion relations of the six modes calculated using the "optical" MDF model with definite symmetry: three antisymmetric (odd) modes $\left(\omega_{1}^{-}\right.$, $\omega_{2}^{-}$, and $\left.\omega_{3}^{-}\right)$and three symmetric (even) modes $\left(\omega_{1}^{+}, \omega_{2}^{+}\right.$, and $\left.\omega_{3}^{+}\right)$. The six dispersion curves agree very well with the loss function intensity patterns, which will help us relate the characteristic structures in the wake potential with the particular modes in the loss function. The loss function is dominated by two highest plasmon-like branches, $\omega_{3}^{ \pm}$, which actually represent modifications of the high-frequency behavior of the Dirac plasmons $\left(\omega_{ \pm}\right)$in Fig. 2(a) by the presence of the $\mathrm{Al}_{2} \mathrm{O}_{3}$ substrate. The odd branch, $\omega_{3}^{-}$, bends toward a finite frequency in the long-wavelength limit $(Q \rightarrow 0)$ due to interaction with the substrate polarization. The four lower branches, $\omega_{1,2}^{ \pm}$, are dominated by the surface phonon modes, as can be concluded from the fact that the two pairs of the surface phonon branches become degenerate at $Q \gtrsim Q_{C}=$ $0.2 \mathrm{~nm}^{-1}$ and they settle at the bulk values of the first and second TO phonon frequencies, $\omega_{1}^{ \pm} \approx \omega_{\mathrm{TO} 1}=48 \mathrm{meV}$ and $\omega_{2}^{ \pm} \approx \omega_{\mathrm{TO} 2}=71 \mathrm{meV}$, respectively. The two faint horizontal lines in Fig. 2(b), which are visible in the loss function at $Q \gtrsim 0.4-0.5 \mathrm{~nm}^{-1}$, represent vertically shifted degenerate pairs of the phonon branches $\omega_{1,2}^{ \pm}$, as a consequence of the nearly static screening by graphene layers [50].

Figure 2(c) shows the loss function in the asymmetric $\operatorname{gr}\left(\right.$ doped) $-\mathrm{Al}_{2} \mathrm{O}_{3}$-gr(undoped) composite. In this system, the Dirac plasmon in doped graphene and the four surface TO phonons in two $\mathrm{Al}_{2} \mathrm{O}_{3}$ hybridize into five modes $\left(\omega_{i}, i=\right.$ $1, \ldots, 5)$ whose dispersion relations are denoted by white dotted lines. In comparison with Fig. 2(b), it is obvious that replacement of the doped by undoped graphene strongly influences the Dirac plasmon intensity. First of all, considering that the undoped graphene does not support Dirac plasmon, the branch $\omega_{3}^{-}$, which is present in Fig. 2(b), disappears in Fig. 2(c). On the other hand, the presence of the undoped graphene practically destroys the plasmonic branch $\omega_{5}$, which appears as $\omega_{3}^{+}$in Fig. 2(b), due to its strong Landau damping by the interband $\pi \leftrightarrow \pi^{*}$ transitions in pristine graphene, in the region $\omega>v_{F} Q$. One further notices that the degeneracy among the four phononic dispersion branches is lifted [50], whereas the intensity of the horizontal line in the loss function 

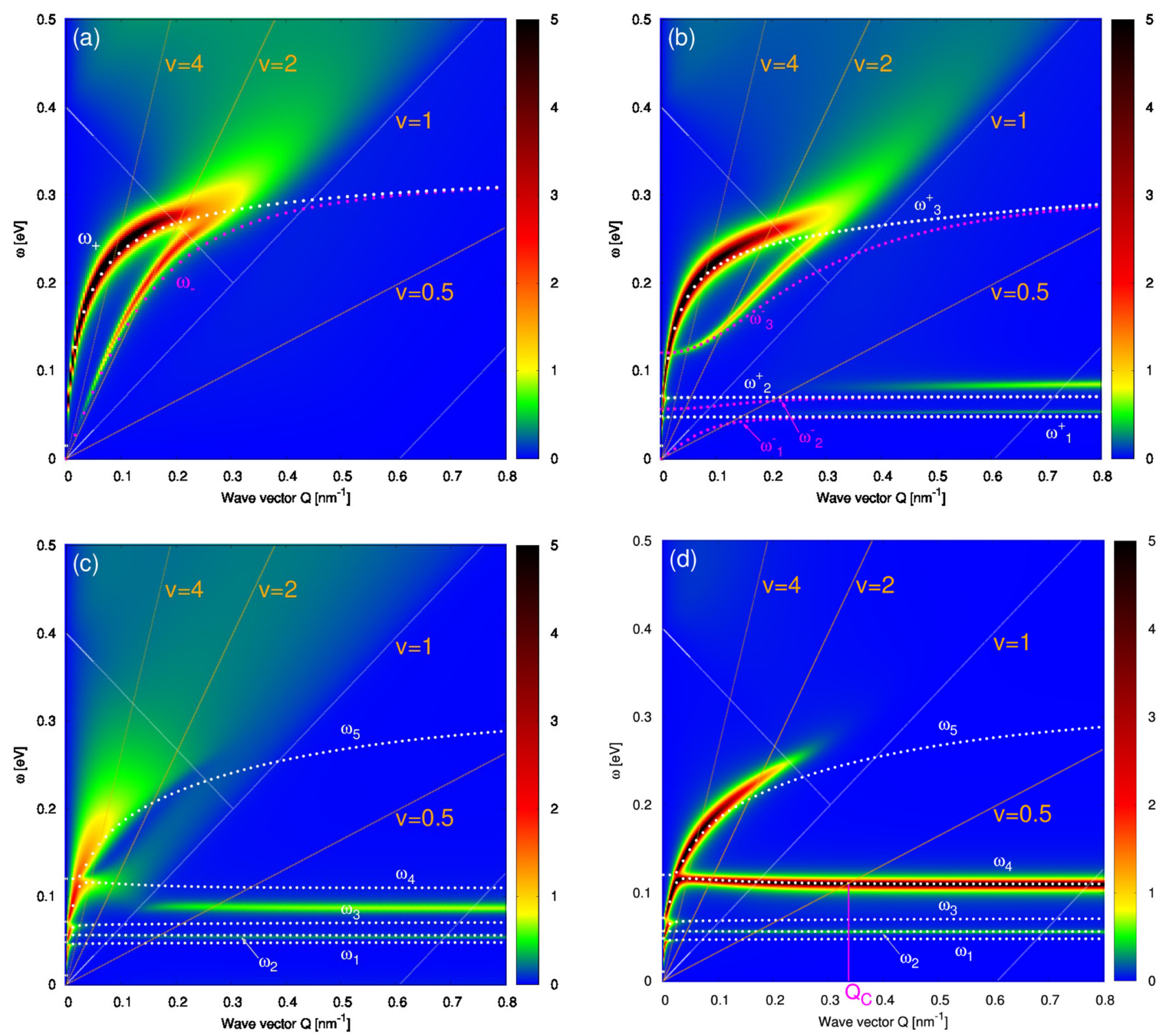

FIG. 2. The loss function in symmetrical (a) gr(doped)-vacuum-gr(doped) and (b) gr(doped)- $\mathrm{Al}_{2} \mathrm{O}_{3}$-gr(doped) composites. The magenta dotted lines show dispersion relations of the odd $\left(\omega_{-}\right)$plasmon-phonon modes, while the white dotted lines show dispersion relations of even $\left(\omega_{+}\right)$plasmon-phonon modes. The loss function in asymmetric (c) gr(doped)- $\mathrm{Al}_{2} \mathrm{O}_{3}$-gr(undoped) and (d) gr(doped)- $\mathrm{Al}_{2} \mathrm{O}_{3}$-vacuum composites. The white dotted lines show dispersion relations of five plasmon-phonon hybridized modes $\left(\omega_{i} ; i=1, \ldots, 5\right)$. The loss function intensities are calculated using the MDF method, while the dispersion relations are obtained from the "optical" MDF method. The separation between graphene layers, or the $\mathrm{Al}_{2} \mathrm{O}_{3}$ slab thickness, is $a=5 \mathrm{~nm}$, and graphene's electronic doping density corresponds to $E_{F}=200 \mathrm{meV}$. The thin white lines show the lower, $\omega=v_{F}\left(Q-2 k_{F}\right)$, and the upper, $\omega=v_{F} Q$, edges of the intraband $\pi^{*} \leftrightarrow \pi^{*}$ electron-hole excitations, as well as the lower edge, $\omega=2 E_{F}-v_{F} Q$, of the interband $\pi \leftrightarrow \pi^{*}$ electron-hole excitations in the Dirac cone approximation. Thin yellow lines denote the $\omega=Q_{x} v$ lines for $v=0.5,1,2$, and 4 in order to visualize their crossing with eigenmodes which contribute in the wake potential.

at large $Q$ values is slightly higher in Fig. 2(c) than in Fig. 2(b).

Figure 2(d) shows the loss function in the asymmetric gr(doped)- $\mathrm{Al}_{2} \mathrm{O}_{3}$-vacuum composite. Similarly to Fig. 2(c), here the Dirac plasmon in doped graphene and the four surface TO phonons in two $\mathrm{Al}_{2} \mathrm{O}_{3}$ hybridize into five modes $\left(\omega_{i}, i=1, \ldots, 5\right)$ whose dispersion relations are denoted by white dotted lines. It should be noted here that the removal of the undoped graphene from the dielectric surface on the right-hand side in Fig. 1 drastically changes the loss function spectral intensity. First of all, because there is no more Landau damping in the region $\omega>v_{F} Q$, the Dirac plasmon $\omega_{5}$ recovers its strength and becomes quite prominent for smaller wave numbers, $Q \lesssim 0.3 \mathrm{~nm}^{-1}$. On the other hand, the higher-lying surface phonon branch at $\omega_{4} \approx 110 \mathrm{meV}$ becomes extremely intense for all wave numbers $Q$ [50]. The latter effect will have significant impact on the wake potential, especially in the low-velocity regime, as will be shown in the next section.

Besides the transitions within and between the conically shaped $\pi$ and $\pi^{*}$ bands, which are included in the MDF 


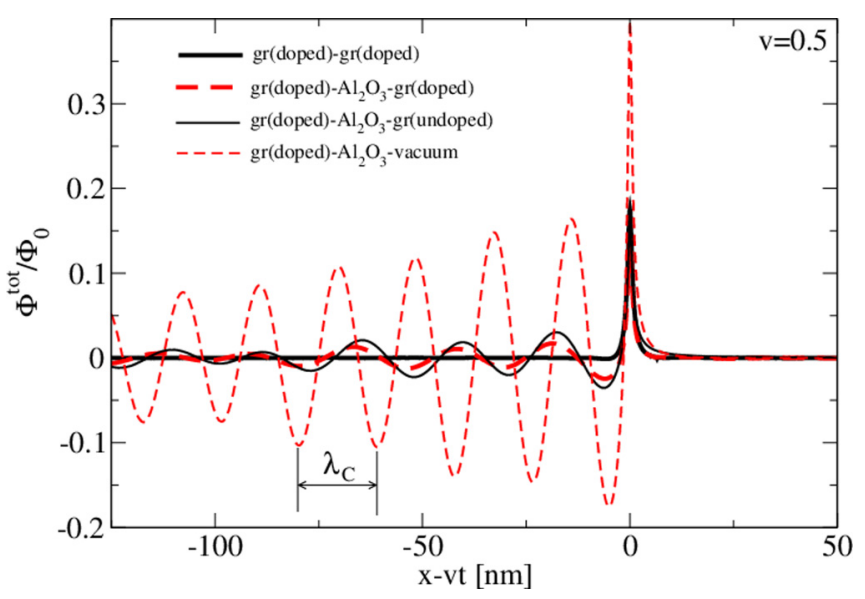

FIG. 3. Wake potential in gr(doped)-gr(doped) (thick black solid line), gr(doped)- $\mathrm{Al}_{2} \mathrm{O}_{3}$-gr(doped) (thick red dashed line), gr(doped)$\mathrm{Al}_{2} \mathrm{O}_{3}$-gr(undoped) (thin black solid line), and $\operatorname{gr}\left(\right.$ doped) $-\mathrm{Al}_{2} \mathrm{O}_{3}$ vacuum (thin red dashed line) composites. The separation between graphene layers is $a=5 \mathrm{~nm}$, the parallel velocity is $v=0.5$, and the height is $z_{0}=5 \AA$.

method, the eHD and ab initio response functions, Eq. (17), also include transitions between more realistically shaped $\pi$ and $\pi^{*}$ bands (including the Van Hove singularities at the $M$ points in the BZ), as well as transitions between higher occupied and unoccupied bands (e.g., $\pi \leftrightarrow \sigma^{*}$ and $\sigma \leftrightarrow \sigma^{*}$ transitions [60]). These transitions represent additional polarization mechanisms that may affect the wake potential in the high-velocity regime, as will be discussed in Sec. III E.

As can be seen from Eq. (1), if the incident particle has the velocity $v$, the only contribution to the wake potential comes from the excitation of polarization modes along the $\omega=Q_{x} v$ line in the $(Q, \omega)$ (quarter) plane. In other words, the charged particle of velocity $v$ can excite all eigenmodes in the system with the phase velocity $\lesssim v$ (or, a particular eigenmode will be excited when the line $\omega=Q_{x} v$ crosses its dispersion relation), giving rise to a steady-state distribution of induced charge, which trails the incident particle. In order to visualize those crossings with eigenmodes, which contribute in the wake potential, we display the $\omega=Q_{x} v$ lines by thin yellow lines in Fig. 2 for $v=0.5,1,2$, and 4 .

\section{Low-velocity limit}

For the low-velocity limit, we consider velocities below the threshold for excitation of Dirac plasmons in doped graphene, $v<1$, in which case the line $\omega=Q_{x} v$ does not cross any of the Dirac plasmonic branches seen in Fig. 2. Results for the wake potential are calculated using the effective dielectric function in Eq. (8) obtained from the MDF method, and they are shown in the reference frame of the moving particle using the normalization by the potential $\Phi_{0}=Z e / z_{0}$.

Figure 3 shows the wake potential in various graphene and $\mathrm{Al}_{2} \mathrm{O}_{3}$ composites, when the charged particle moves at a distance $z_{0}=5 \AA$ with a velocity $v=0.5$, while the thickness of the structure is $a=5 \mathrm{~nm}$. The wake potential is plotted as a function of $x$ for $y=0$ in the $z=a / 2$ plane. The thick black solid line shows the total potential in the gr(doped)-vacuum-gr(doped) structure, exhibiting only a sharp peak around the charged particle position $(x=v t)$ in violation of the perfect screening regime; namely, the potential produced by the charged particle at the $z=a / 2$ plane in free space $\left(Z e / z_{0}\right)$ is not perfectly compensated by the potential produced by its image charge $\left(-Z e / z_{0}\right)$. In this case, the incident particle does not drag waves behind itself because its velocity is always below the phase velocities of the two hybridized Dirac plasmons, as can be seen in Fig. 2(a).

The thick red dashed line in Fig. 3 shows the wake potential when $\mathrm{Al}_{2} \mathrm{O}_{3}$ is inserted between two doped graphene layers, i.e., in the system $\operatorname{gr}($ doped $)-\mathrm{Al}_{2} \mathrm{O}_{3}$-gr(doped). In this structure, the charged particle drags relatively weak polarization waves. This is because this system supports two low-intensity TO phonon branches, which are crossed by the $v=0.5$ line, as can be seen in Fig. 2(b). The thin black line in Fig. 3 represents the wake potential in the $\operatorname{gr}\left(\right.$ doped) $-\mathrm{Al}_{2} \mathrm{O}_{3}$-gr(undoped) composite. The graphene layer closer to the charged particle is now undoped (and hence it does not support Dirac plasmon), so the screening of the TO phonons in the $\mathrm{Al}_{2} \mathrm{O}_{3}$ surface is weaker, giving rise to slightly stronger oscillations in the wake potential. This is also evident from Fig. 2(c), where it can be seen that the $v=0.5$ line crosses the horizontal phonon line in the loss function, which is much stronger than the corresponding phonon line in Fig. 2(b). The thin red dashed line in Fig. 3 shows the wake potential in the gr(doped)- $\mathrm{Al}_{2} \mathrm{O}_{3}$-vacuum composite. Now, the wake potential is strongly enhanced in comparison with the other cases. This is because the removal of the graphene layer closer to the incident particle leaves the electrical field, which excites the surface $\mathrm{TO}$ phonons in the $\mathrm{Al}_{2} \mathrm{O}_{3}$ layer, fully unscreened. It should be recalled from Fig. 2(d) that the intensity of the phonon branch $\omega_{4}$ (which is crossed by the $v=0.5$ line) is very strong, resulting in a strong enhancement of oscillations in the wake potential.

Therefore, in the velocity range $v<1$, the oscillations in the wake potential come from excitations of the surface TO phonons, with an amplitude that depends on how the TO phonons are screened by graphene adlayers. It can be seen that a characteristic wavelength of oscillations in the wake potential can be determined from a critical wave number for which the $\omega=Q v$ line crosses the most intense among the phononic dispersion relations,

$$
\lambda_{C}=\frac{2 \pi}{Q_{C}} .
$$

For example, this can be tested for the case when the $\omega=$ $Q v$ line crosses the intense $\omega_{4}$ branch. As denoted by the magenta line in Fig. 2(d), it occurs for $Q_{C}=0.34 \mathrm{~nm}^{-1}$, which from Eq. (25) gives $\lambda_{C}=18.5 \mathrm{~nm}$. On the other hand, the wavelength determined directly from Fig. 3 (which is indicated in the graph) is $\lambda_{C}=18.9 \mathrm{~nm}$.

We did not explore the wake potential in the plane $z=$ $-a / 2$ of the composite with various graphene/vacuum configurations, i.e., on the side opposite from the incident particle, for the following reason. Namely, at low velocities, the $\omega=$ $Q v$ line crosses the surface phonon branches at larger wave numbers, $Q \approx \omega_{i} / v$, implying that the factor $e^{-2 Q a}$, which controls interaction between the phonon modes in the left and right surfaces of the dielectric layer in Fig. 1, is small enough 

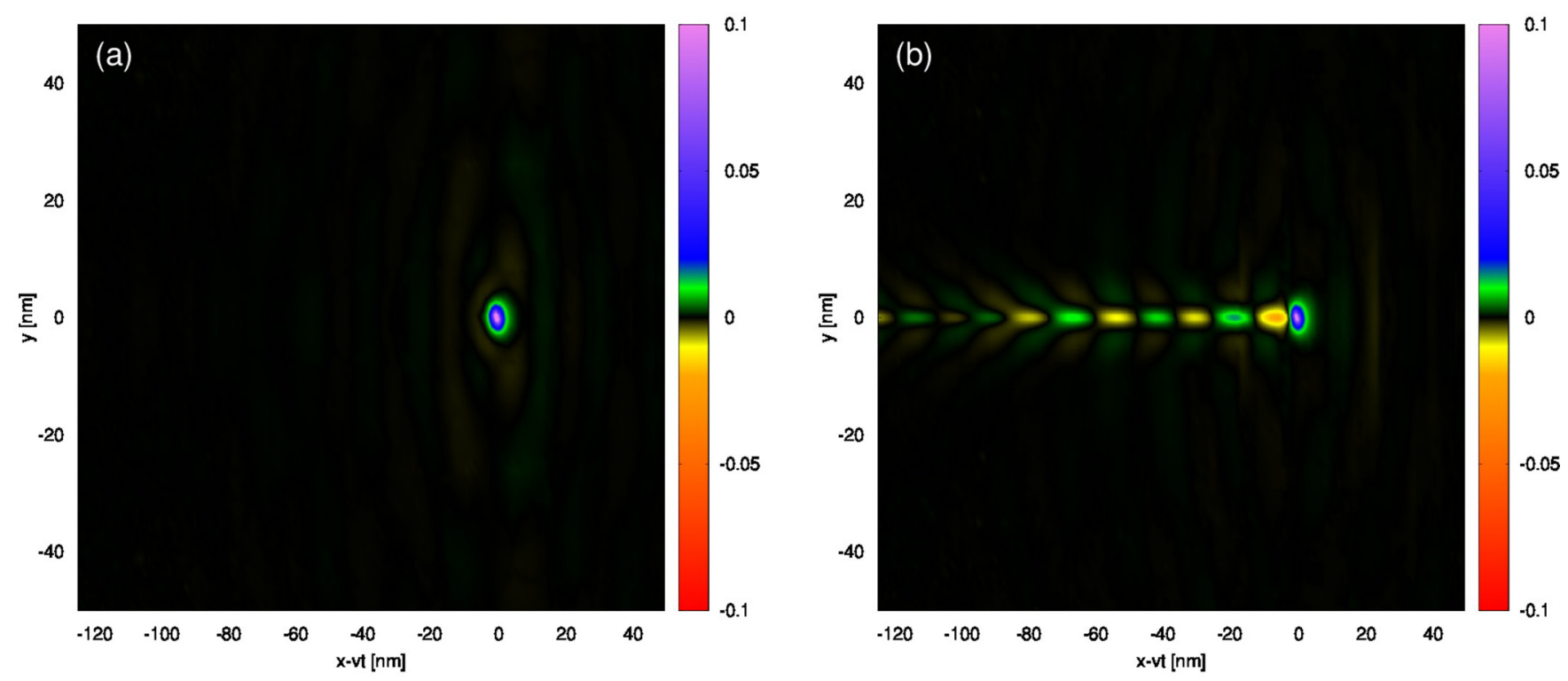

FIG. 4. The distribution of the wake potential in the plane $z=a / 2$ for (a) $\operatorname{gr}\left(\right.$ doped)-gr(doped) and (b) $\operatorname{gr}(\mathrm{doped})$ - $\mathrm{Al}_{2} \mathrm{O}_{3}$-gr(doped) composites. The parameters are the same as in Fig. 3.

to render the wake potential in the plane $z=-a / 2$ heavily suppressed. For the same reason, the wake potential in the plane $z=a / 2$, lying closer to the incident particle, is almost independent from the polarization that is localized in the plane $z=-a / 2$ on the opposite side of the structure. Therefore, the moving particle interacts predominantly with phonon modes in the nearest surface, so that the structure may be effectively considered as the surface of a semi-infinite dielectric, which is either free or covered by a single layer of graphene. As we shall see later, this will not be the case for intermediate and high velocities, $v>1$.

In order to explore the spatial distribution of the wake patterns, we show in Fig. 4 the wake potential across the $z=a / 2$ plane, using the same parameters as in Fig. 3. If the charged particle moves above two doped graphene layers, the wake potential, shown in Fig. 4(a), only consists of an anisotropic narrow peak located around $x=v t$. On the other hand, if the $\mathrm{Al}_{2} \mathrm{O}_{3}$ slab is inserted between two doped graphene layers, the wake potential, shown in Fig. 4(b), develops waves behind the moving particle. Those waves are the signature of the surface phonons, which are dragged by the charged particle, as discussed in Fig. 3. What is intriguing here is the fact that the potential is very narrowly localized around the particle trajectory $(x=v t, y=0)$ without any noticeable $\mathrm{V}$-shaped spreading. This narrow distribution of the wave pattern seems to be a signature of the phonon-dominated wake effect [25].

\section{Intermediate velocities}

We next consider velocities above the threshold for excitation of Dirac plasmons, which possess phase velocities that are always greater than the Fermi velocity in graphene, $v_{F}=1$ (in our units), as can be seen in Fig. 2.

Figure 5 shows the wake potential in various $\mathrm{sy}_{1}-\mathrm{Al}_{2} \mathrm{O}_{3}-\mathrm{sy}_{2}$ composites of thickness $a=5 \mathrm{~nm}$ with the charged particle moving at the height $z_{0}=5 \AA$, whereas for its velocity we chose $v=2$. The wake potential is first plotted as a function of $x$ for $y=0$ in the plane $z=a / 2$, as before. Calculations are performed using the MDF method.

Figure 5(a) displays the same graphene/vacuum configurations on the right-hand side of the composite structure as shown in Fig. 3. In the case of two doped graphene layers with vacuum between them (thick black solid line), the wake potential exhibits oscillations, which trail behind the charged particle. Those oscillations represent polarization waves resulting from excitations of the two hybridized Dirac plasmons, as can be seen in Fig. 2(a) where the $v=2$ line crosses the dispersion curves of those plasmons. When an $\mathrm{Al}_{2} \mathrm{O}_{3}$ slab is inserted between two doped graphene layers (red dashed line), the first two oscillations behind the particle in the wake potential become slightly suppressed. This is because the Dirac plasmons hybridize with the surface TO phonons, so that the lower Dirac plasmon branch $\omega_{3}^{-}$starts at a finite frequency for $Q \rightarrow 0$. Accordingly, the $\omega=Q v$ line crosses the lower Dirac plasmon branch at a smaller $Q_{C}$, where this mode has lower intensity, as can be seen by a comparison of Figs. 2(a) and 2(b). When the doped graphene on the right-hand side of the structure is replaced by an undoped graphene (thin black solid line), oscillations from two strongly interacting Dirac plasmons disappear, and only a very weak and rather long-ranged wave remains behind the charged particle. This occurs because the undoped graphene does not support Dirac plasmons [the plasmon branch $\omega_{3}^{-}$, present in Fig. 2(b), now disappears], while the remaining Dirac plasmon in the doped graphene on the left-hand side [the plasmon branch $\omega_{5}$ in Fig. 2(c)] becomes strongly Landau damped by the interband $\pi \leftrightarrow \pi^{*}$ transitions in the undoped graphene on the right-hand side. As a result, the $v=2$ line crosses the already very weakened and broad Dirac plasmon [labeled by $\omega_{5}$ in Fig. 2(c)], so that fast oscillations disappear from the wake potential. On the other hand, the undoped graphene also strongly screens the TO surface phonons, which results in very weak oscillations with long wavelengths in the wake potential. This scenario is consistent with the strong 

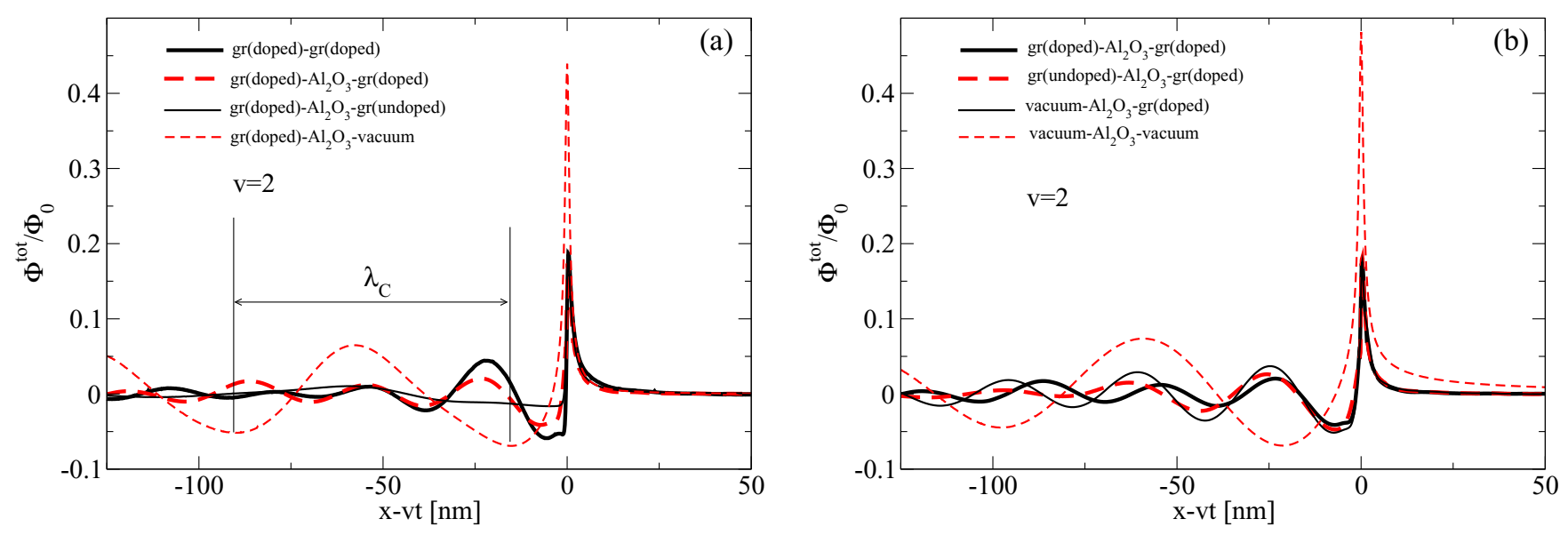

FIG. 5. (a) Wake potential in gr(doped)-gr(doped) (thick black solid line), gr(doped)- $\mathrm{Al}_{2} \mathrm{O}_{3}$-gr(doped) (thick red dashed line), gr(doped)$\mathrm{Al}_{2} \mathrm{O}_{3}$-gr(undoped) (thin black solid line), and gr(doped) $-\mathrm{Al}_{2} \mathrm{O}_{3}$-vacuum (thin red dashed line) composites. (b) Wake potential in gr(doped)$\mathrm{Al}_{2} \mathrm{O}_{3}$-gr(doped) (thick black solid line), gr(undoped)- $\mathrm{Al}_{2} \mathrm{O}_{3}$-gr(doped) (thick red dashed line), vacuum- $\mathrm{Al}_{2} \mathrm{O}_{3}$-gr(doped) (thin black solid line), and vacuum- $\mathrm{Al}_{2} \mathrm{O}_{3}$-vacuum (thin red dashed line) composites. The separation between graphene layers or the $\mathrm{Al}_{2} \mathrm{O}_{3}$ thickness is $a=5 \mathrm{~nm}$, the parallel velocity is $v=2$, and the height is $z_{0}=5 \AA$.

enhancement of oscillations in the wake potential, which occurs when the undoped graphene is removed from the right-hand side of the structure, as shown by the thin red dashed line in Fig. 5(a). In this configuration, the $\omega=Q v$ line crosses the strong (unscreened) surface TO phonon mode $\omega_{4}$, but it also crosses the Dirac plasmon $\omega_{5}$, which recovers its intensity when the undoped graphene is removed, as can be seen in Fig. 2(d). Accordingly, one would expect that the wake oscillations in the thin red dashed line also contain a component with a wavelength that comes from the excitation of the Dirac plasmon in doped graphene on the left-hand side of the structure. However, because the wavelength value of $\lambda_{C}=72 \mathrm{~nm}$, obtained using Eq. (25) and the wave number $Q_{C}=0.087 \mathrm{~nm}^{-1}$ at which the $v=2$ line crosses the $\omega_{4}$ branch in Fig. 2(d), agrees very well with the wavelength $\lambda_{C} \approx 75 \mathrm{~nm}$ taken directly from Fig. 5(a), it appears that the surface TO phonon mode with $\omega_{4}$ provides a dominant contribution to the wake potential in this configuration.

In Fig. 2, one can see that when the velocity takes the value $v=2$, the dispersion curves for all eigenmodes are crossed by the line $\omega=Q v$ at wave numbers $Q<0.2 \mathrm{~nm}^{-1}$. Considering that the slab thickness is $a=5 \mathrm{~nm}$, the interaction between eigenmodes that are localized on the surfaces on the left-hand side and the right-hand side of the structure is governed by a factor $e^{-2 Q a}>e^{-2}$, which is sufficiently large to allow for strong hybridization to take place among those modes. Therefore, the wake potential in the $z=a / 2$ plane should be sensitive to the configuration of the surface in the $z=-a / 2$ plane on the opposite side of the structure. To explore this aspect of the problem, we use in Fig. 5(b) the thick black solid, thick red dashed, and thin black lines to show the wake potential in the $\operatorname{gr}$ (doped)- $\mathrm{Al}_{2} \mathrm{O}_{3}-\mathrm{gr}$ (doped), gr(undoped)- $\mathrm{Al}_{2} \mathrm{O}_{3}$-gr(doped), and vacuum- $\mathrm{Al}_{2} \mathrm{O}_{3}$-gr(doped) composites, respectively. By the comparison of the thick red dashed curves in Figs. 5(a) and 5(b), it can be seen that when the doped graphene on the left-hand side is replaced by an undoped graphene, the wake potential changes, but not drastically. Referring to Fig. 2(b), this replacement causes the disappearance of the $\omega_{3}^{-}$mode, so that only the plasmonic $\omega_{3}^{+}$mode contributes to the wake potential. This is probably manifested as a slight phase shift in the oscillatory pattern, in comparison with the case when two Dirac plasmons contribute. Also, the interband $\pi \leftrightarrow \pi^{*}$ transitions in the undoped graphene broaden the $\omega_{3}^{+}$plasmon mode due to Landau damping, so that the wake potential becomes weaker in the region far from the particle. When the undoped graphene is removed from the left-hand side of the structure, the interband $\pi \leftrightarrow \pi^{*}$ transitions disappear, and the Dirac plasmon in the doped graphene on right-hand side recovers its strength, so that the wake potential becomes enhanced, as evidenced by the thin black line in Fig. 5(b). Finally, removal of both graphene layers from the structure unscreens the electric field, which excites the surface TO phonons [similarly to the branch $\omega_{4}$ in Fig. 2(d)], so the oscillations in the wake potential are strongly enhanced, as shown by the thin red dashed line in Fig. 5(b). A similar effect occurs when just the graphene layer on the right-hand side is removed from the plane closer to the charged particle, as already shown by the thin red dashed line in Fig. 5(a).

In Fig. 6(a), we show spatial distribution of the wake potential across the $z=a / 2$ plane in a gr(doped)-vacuumgr(doped) composite, where the separation between the graphene layers is $a=5 \mathrm{~nm}$, the parallel velocity of the particle is $v=2$, and its height is $z_{0}=5 \AA$. The wavelength of the polarization waves lagging behind the particles is $\lambda_{C}=$ $32 \mathrm{~nm}$, which agrees with the wavelength $\lambda_{C}=30 \mathrm{~nm}$ obtained using Eq. (25) and the wave number $Q_{C}=0.21 \mathrm{~nm}^{-1}$ for which the $v=2$ line crosses the even Dirac plasmon branch $\omega_{+}$in Fig. 2(a). This undoubtedly proves that the main contribution to the wake potential in this configuration comes from the even plasmon with $\omega_{+}$. A deviation from an ideally filled $\mathrm{V}$ shape is evident in Fig. 6(a) due to the appearance of a "hole" in the sector near $x=-70 \mathrm{~nm}$. This "hole" is probably a consequence of a small contribution from the odd $\omega_{-}$plasmon in the wake potential, which causes a locally destructive interference with the main oscillations coming from the $\omega_{+}$mode.

Figure 6(b) shows the spatial distribution of the wake potential in the $\operatorname{gr}\left(\right.$ doped) $-\mathrm{Al}_{2} \mathrm{O}_{3}$-gr(doped) composite. From 

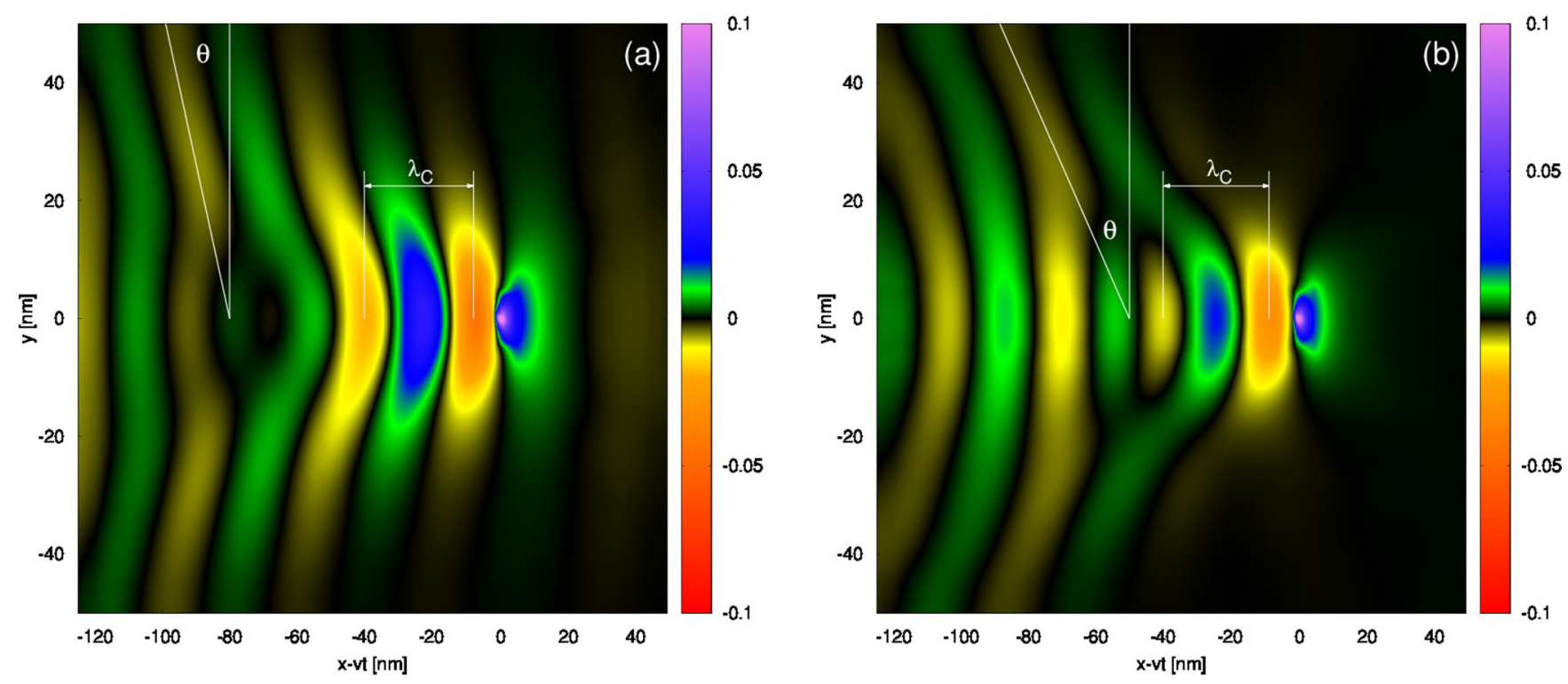

FIG. 6. The distribution of the wake potential in the plane $z=a / 2$ for (a) $\operatorname{gr}\left(\right.$ doped)-gr(doped) and (b) $\operatorname{gr}(\mathrm{doped})-\mathrm{Al}_{2} \mathrm{O}_{3}-\operatorname{gr}(\mathrm{doped})$ composites. The separation between graphene layers is $a=5 \mathrm{~nm}$, the parallel velocity is $v=2$, and the height is $z_{0}=5 \AA$.

Fig. 2(b) is evident that for $v=2$, the $\omega=Q v$ line does not cross any prominent phonon branches, and therefore phonons are not responsible for the features in the wake potential in Fig. 6(b); i.e., here the hybridized Dirac plasmons with $\omega_{3}^{ \pm}$ are still the main generators of those features. The dielectric layer reduces the amplitude of the wake potential in the sector just behind the charged particle. This is because the dielectric layer screens the intensity of hybridization among the Dirac plasmons, changing the modes $\omega_{ \pm}$seen in Fig. 2(a) to the modes $\omega_{3}^{ \pm}$in Fig. 2(b). In addition, the presence of the TO phonon modes in the dielectric bends the $\omega_{3}^{-}$plasmon towards a finite frequency when $Q \rightarrow 0$, so that the $\omega=Q v$ line crosses the curve $\omega_{3}^{-}$at a smaller $Q_{C}$ than in the case of its crossing the plasmon with $\omega_{-}$. Consequently, a contribution from the $\omega_{3}^{-}$plasmon distorts the "ideal" $V$ shape in the sector near $x=-50 \mathrm{~nm}$, in a manner that is different from the "hole" due to the $\omega_{-}$plasmon, seen in Fig. 6(a). It may also be noticed from both panels in Fig. 6 that the wave-front angle $\theta$ of the $\mathrm{V}$ shapes increases when the dielectric is inserted between the graphene layers.

\section{E. High-velocity limit}

When the velocity $v$ further increases beyond the value $v=$ 2 , the wave number $Q_{C}$ at which the $\omega=Q v$ line crosses the Dirac plasmons decreases; i.e., the characteristic wavelength $\lambda_{C}$ of the wake potential increases. We explore this here using calculations based on the MDF method.

Figure 7 shows the wake potential in the gr(doped)vacuum-gr(doped) (black solid line) and in the gr(doped)$\mathrm{Al}_{2} \mathrm{O}_{3}$-gr(doped) (red dashed line) composites when the incident particle velocity is $v=4$. As can be seen in Fig. 2(a), the $\omega=Q v$ line does not cross the $\omega_{-}$branch for $v=4$, so the only contribution to the wake potential in this configuration is expected to come from the excitation of the plasmon $\omega_{+}$mode. As a result, the wake potential becomes an almost perfect "single-harmonic" wave, which lags behind the charged particle. The wavelength $\lambda_{C}=66 \mathrm{~nm}$ taken from Fig. 7 agrees well with $\lambda_{C}=69 \mathrm{~nm}$ obtained from Eq. (25), and the wave number $Q_{C}=0.09 \mathrm{~nm}^{-1}$ for which the $v=4$ line crosses the even Dirac plasmon branch $\omega_{+}$in Fig. 2(a). When the dielectric layer is inserted between graphene layers, the $\omega_{3}^{-}$bends toward a finite energy for $Q \rightarrow 0$, and the line $\omega=Q v$ crosses it, but there is a relatively minor change in the shape of the wake potential. In the case without the dielectric layer, one sees a longer-ranged bow wave in front of the particle, as well as a narrow dip right behind the particle, which disappears upon the inclusion of the dielectric layer. At longer distances behind the particle, one sees in Fig. 7 that the main modification of the wake potential is a slight increase of the wavelength to the value $\lambda_{C}=72 \mathrm{~nm}$ in the case with the dielectric layer. This is because the dielectric screening

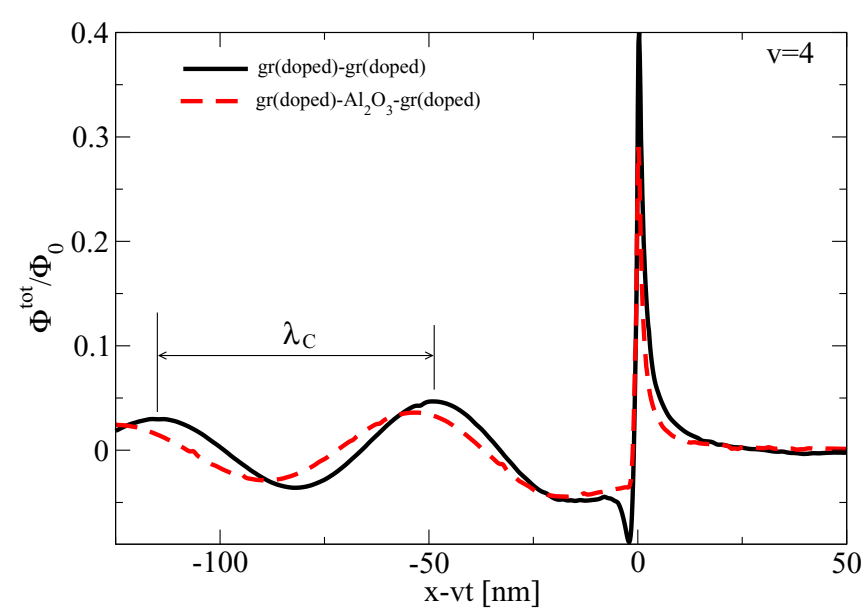

FIG. 7. Wake potential in gr(doped)-gr(doped) (black solid line) and $\operatorname{gr}$ (doped) $-\mathrm{Al}_{2} \mathrm{O}_{3}$-gr(doped) (red dashed line) composites. The separation between graphene layers is $a=5 \mathrm{~nm}$, the parallel velocity is $v=4$, and the height is $z_{0}=5 \AA$. 

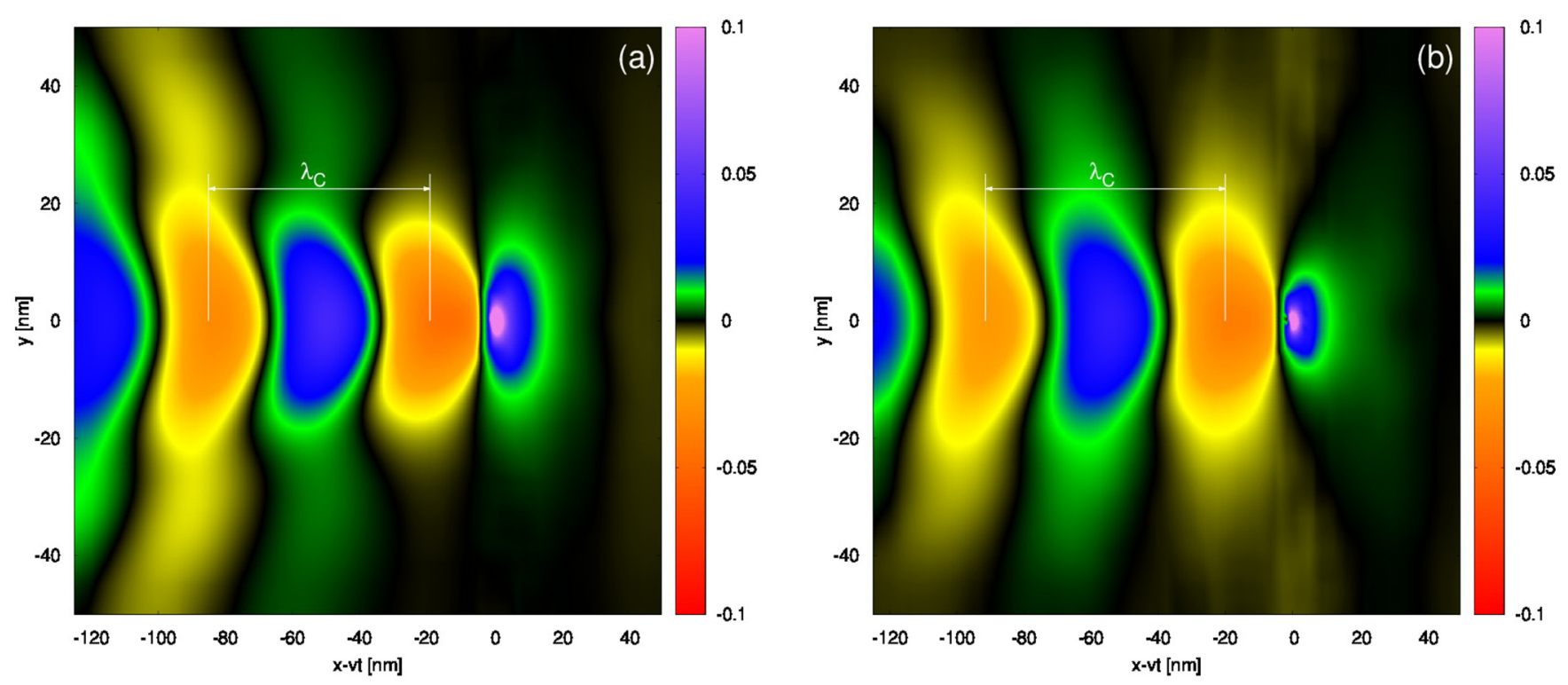

FIG. 8. The distribution of the wake potential in the plane $z=a / 2$ for (a) $\operatorname{gr}\left(\right.$ doped)-gr(doped) and (b) $\operatorname{gr}(\mathrm{doped})-\mathrm{Al}_{2} \mathrm{O}_{3}-\mathrm{gr}(\mathrm{doped})$ composites. The separation between graphene layers or the $\mathrm{Al}_{2} \mathrm{O}_{3}$ slab thickness is $a=5 \mathrm{~nm}$, the parallel velocity is $v=4$, and the height is $z_{0}=5 \AA$.

slightly shifts the $\omega_{+}$branch toward lower energies, so the line $\omega=Q v$ crosses it at a somewhat smaller $Q_{C}$, as can be seen in Fig. 2(b).

Figure 8(a) shows a planar distribution of the wake potential for the $\operatorname{gr}$ (doped)-vacuum-gr(doped) composite. In the region $|y| \lesssim 20 \mathrm{~nm}$, the wake potential behaves as a welldeveloped $\mathrm{V}$ shape lagging behind the particle. However, in the region $|y| \gtrsim 20 \mathrm{~nm}$, one can notice an unusual addition to this primary $\mathrm{V}$ shape in the form of a fainter secondary $\mathrm{V}$ shape with a larger opening angle than the angle of the primary $\mathrm{V}$ shape. This broad, secondary $\mathrm{V}$ shape could be attributed to a nonresonant (reactive) contribution of the $\omega_{-}$ plasmon to the wake potential. Figure 8(b) shows modifications in the planar wake potential occurring when the dielectric $\mathrm{Al}_{2} \mathrm{O}_{3}$ is inserted between graphene layers. As already pointed out, the even $\omega_{3}^{-}$branch bends toward a finite energy [see Fig. 2(b)] and the $\omega=Q v$ line crosses it, but the primary $\mathrm{V}$ shape in the wake potential in the region $|y| \lesssim 20 \mathrm{~nm}$ does not change very much. We can notice a slight increase of the wavelength $\lambda_{C}$ in the primary $\mathrm{V}$ shape, as already discussed in Fig. 7. Probably the main effect of the $\omega=Q v$ line crossing the $\omega_{3}^{-}$branch in the presence of the dielectric layer is a modification of the wake potential in the region $|y| \gtrsim 20 \mathrm{~nm}$ in Fig. 8(b), which contains a broader distribution of the wave fronts at distances $-40 \mathrm{~nm} \lesssim x-v t<0$ behind the particle than in the secondary $\mathrm{V}$ shape in Fig. 8(a).

\section{F. Wake potential from $a b$ initio and eHD methods}

As already discussed, when the dominant features in the wake potential are governed by the hybridized Dirac plasmon-TO phonon modes in the $\mathrm{THz}$ to the mid-IR frequency range, it is sufficient to use the MDF method in calculations, which captures all those features. Indeed, we have verified that using the $a b$ initio method instead of the MDF method does not change the qualitative and quantitative picture of the wake potential for low and intermediate velocities. However, for larger velocities, the $\omega=$ $Q v$ line starts to also cross the high-energy $\pi$ plasmons in graphene, which happens at small enough wave numbers $Q_{\pi}$, where the $\pi$ plasmon represents a sufficiently strong dipolar mode, which could introduce some new features in the wake potential. In order to capture those features, one should use the $a b$ initio method, which is expected to yield accurate enough results in its optical limit. Alternatively, one can simply use the eHD method to get a qualitatively correct account of the high-energy $\pi \rightarrow \pi^{*}$ interband transitions near the $M$ points in the BZ of graphene, occurring at $\omega \approx 4 \mathrm{eV}$.

In order to facilitate our analysis of contributions from various modes in the wake potential, we let $a \rightarrow \infty$, so that the structure is represented by a semi-infinite dielectric with a single layer of graphene on top of it, which can support at most three hybridized Dirac plasmon-TO phonon modes plus the high-energy $\pi$ plasmon.

Figure 9 shows the loss function obtained using the $a b$ initio method in an extended $(Q, \omega)$ phase space for two configurations: (a) $\mathrm{Al}_{2} \mathrm{O}_{3}$ (semi-infinite)-gr(doped) and (b) $\mathrm{Al}_{2} \mathrm{O}_{3}$ (semi-infinite)-gr(undoped) composites. The loss function spectra with doped graphene exhibit two main features: a narrow and intense low-energy Dirac plasmon, which occupies only a very small part of the $(Q, \omega)$ phase space, but gives a very important contribution to the wake potential, and a very broad, intense $\pi$ plasmon, which should also give a significant contribution to the wake potential in the high-velocity limit. On the other hand, the loss function for the structure with undoped graphene is only dominated by the broad $\pi$ plasmon. In all the spectra, there exist barely noticeable lowenergy $(\omega \sim 100 \mathrm{meV}$ ) horizontal TO phonon branches.

Figure 10 shows the wake potential in (a) $\mathrm{Al}_{2} \mathrm{O}_{3}$ (semiinfinite)-gr(doped) and (b) $\mathrm{Al}_{2} \mathrm{O}_{3}$ (semi-infinite)-gr(undoped) composites obtained using the $a b$ initio method (black solid lines in both panels) and the eHD method [magenta dashed 

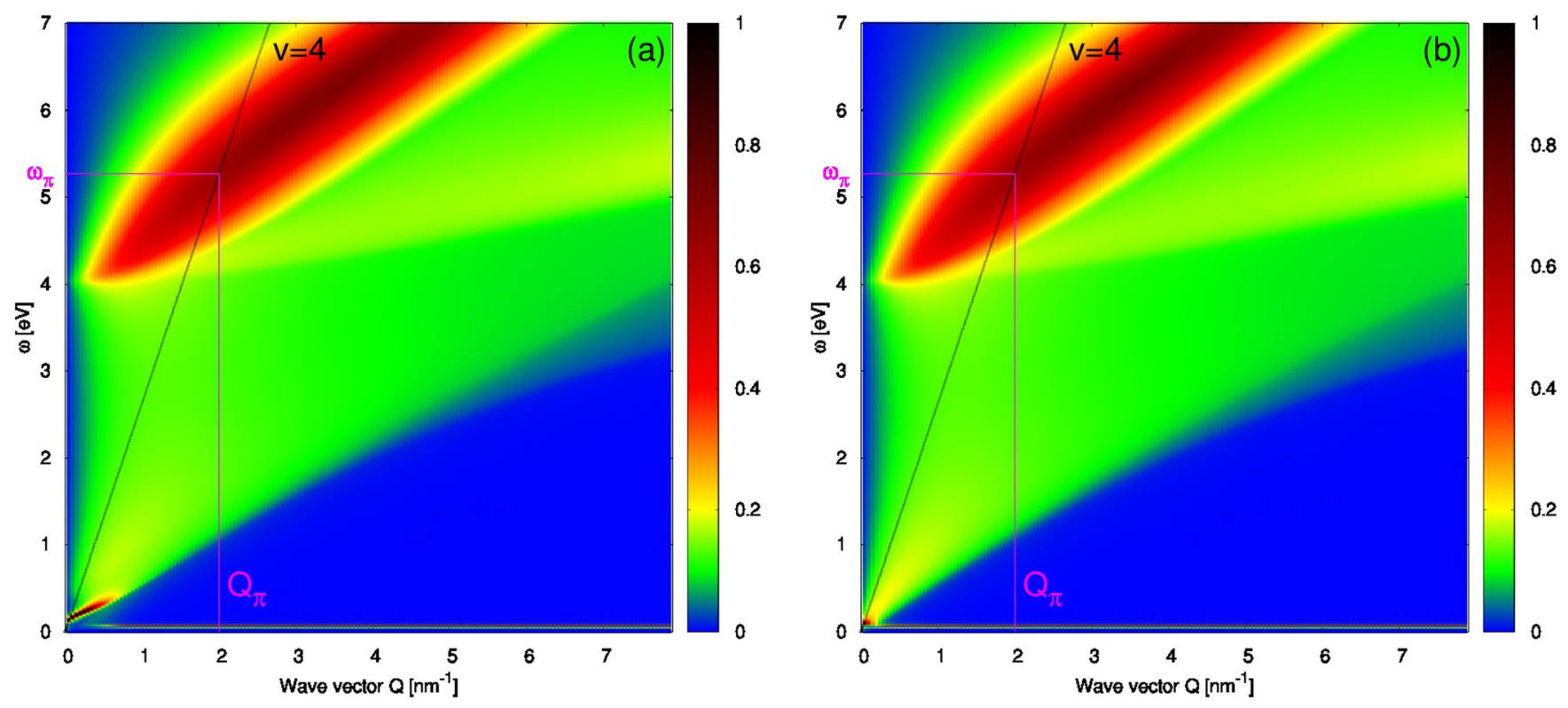

FIG. 9. The loss function in (a) $\mathrm{Al}_{2} \mathrm{O}_{3}$ (semi-infinite)-gr(doped) and (b) $\mathrm{Al}_{2} \mathrm{O}_{3}$ (semi-infinite)-gr(undoped) composites obtained using the ab initio method. The $Q_{\pi}$ and $\omega_{\pi}$ represent the wave vector and frequency, respectively, for which the $v=4$ line crosses the centroid of the $\pi$ plasmon.

line in panel (b)]. The case with doped graphene shows slow oscillations with $\lambda_{C} \approx 92.6 \mathrm{~nm}$ in Fig. 10(a) that corresponds

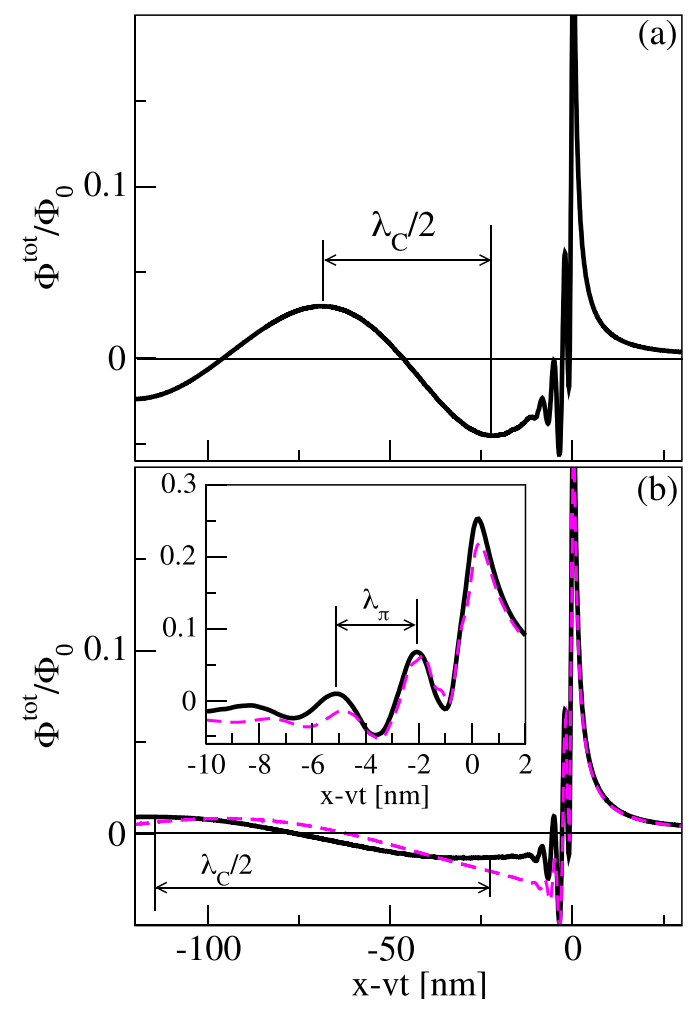

FIG. 10. The wake potential in (a) $\mathrm{Al}_{2} \mathrm{O}_{3}$ (semi-infinite)gr(doped) and (b) $\mathrm{Al}_{2} \mathrm{O}_{3}$ (semi-infinite)-gr(undoped) composites obtained using the $a b$ initio method (black solid line) and the eHD method (magenta dashed line). The charged particle velocity is $v=4$ and the height is $z_{0}=5 \AA$. The inset shows the zoom of panel (b) in the region $x \lesssim v t$ in order to emphasize the contribution of the $\pi$ plasmon to the wake potential. to the excitation of the Dirac plasmon. Besides those slow oscillations, one can notice very fast (noise-like) oscillations in the region just behind the charged particle $(x \lesssim v t)$. The same oscillations also occur in Fig. 10(b) when the doped graphene is replaced by an undoped graphene, where they are accompanied by rather weak and very slow oscillations with $\lambda_{C} \approx 180 \mathrm{~nm}$, corresponding to the excitations of the TO phonon modes at very short wave numbers. In order to explore the origin of those fast oscillations, the inset to Fig. 10(b) shows a zoomed-in portion of the wake potential in the range $-10 \mathrm{~nm}<x-v t<2 \mathrm{~nm}$. Obviously, the fast oscillations are not noise, but rather have a well-defined wavelength $\lambda_{\pi}$. Comparing the value $\lambda_{\pi}=3.1 \mathrm{~nm}$ obtained from Eq. (25) and the wave number $Q_{\pi}=2 \mathrm{~nm}^{-1}$ for which the $v=4$ line crosses the $\pi$ plasmon in Fig. 9 with the wavelength of the fast oscillations $\lambda \approx 3.0 \mathrm{~nm}$ obtained directly from the inset to Fig. 10(b), we may conclude that those fast oscillations represent the wake pattern due to excitation of the $\pi$ plasmon.

It is interesting to note that the eHD method (magenta line), in which transitions between the $\pi$ and $\pi^{*}$ bands at the $M$ points of the $\mathrm{BZ}$ are included with a few parameters in our simple eHD model for the optical conductivity of graphene, Eq. (12) [57,58], is capable of providing a very good agreement with the ab initio results, especially at distances close to the charged particle. This is particularly remarkable given the fact that the eHD method is formulated in the optical limit; see Eq. (9).

Figure 11 shows the spatial distribution of the wake potential in the plane $z=a / 2$ in the $\mathrm{Al}_{2} \mathrm{O}_{3}$ (semi-infinite)gr(undoped) composite obtained using the $a b$ initio method. The charged particle velocity is $v=4$ and its height is $z_{0}=$ $5 \AA$. In spite of a diffuse background behind the charged particle, which is likely coming from the large width of the $\pi$ plasmon and/or from the extended continuum of the electron-hole excitations at $\omega>Q v_{F}$ in Fig. 9(b), one can see 


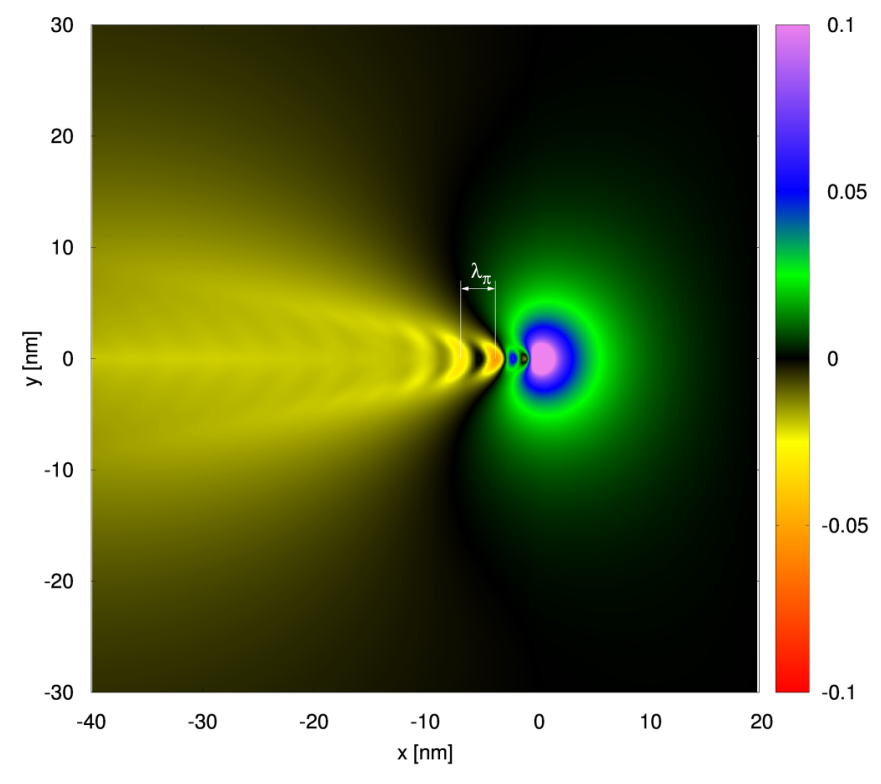

FIG. 11. The distribution of the wake potential in the plane $z=$ $a / 2$ for $\mathrm{Al}_{2} \mathrm{O}_{3}$ (semi-infinite)-gr(undoped) composite obtained using the $a b$ initio method. The charged particle velocity is $v=4$ and the height is $z_{0}=5 \AA$.

that a well-developed V-shaped pattern emerges in Fig. 11, containing waves of the wavelength $\lambda_{\pi}$ that lag behind the charged particle, which represent the $\pi$ plasmon in graphene. This is a nice verification of the fact that for larger wave numbers (above the optical regime), $Q \sim 2 \mathrm{~nm}^{-1}$ ( $\sim 0.1$ a.u.), electron transitions between the $\pi$ and $\pi^{*}$ bands at the $M$ points indeed constitute a collective mode, or plasmon, as predicted in Ref. [60].

\section{CONCLUSIONS}

We have performed a detailed study of the wake potential in various $\mathrm{sy}_{1}-\mathrm{Al}_{2} \mathrm{O}_{3}-\mathrm{sy}_{2}$ composites, where $\mathrm{sy}_{i}$ with $i=1,2$ may be vacuum, pristine graphene, or doped graphene, by considering three velocity regimes using three complementary methods for the response function of graphene. It was found that the wake potential calculated using the ab initio method agrees very well with the results obtained using the MDF method at low and intermediate velocities for both doped and undoped graphene layers, and it agrees well with the results obtained using the eHD method at large velocities for undoped graphene.

It was shown that in the low-velocity regime (below the threshold for excitation of the Dirac plasmon in graphene, $v<$ $1)$, the charged particle excites only the polarization modes in the adjacent surface (nearest to the charged particle). In this regime, it is the excitation of the TO phonons in the dielectric layer that gives a dominant contribution to the wake potential, which is strongly dampened when the adjacent dielectric surface is covered by graphene. It was shown that the spatial distribution of this phonon-dominated wake pattern is narrowly localized along the line parallel to the incident particle trajectory.

For intermediate velocities (above the threshold for excitations of the Dirac plasmons, $v>1$ ), the hybridized plasmon-
TO phonon modes that are localized on the opposite surfaces of the structure contribute to the wake potential in the adjacent surface. In this regime, the dominant contribution comes from the hybridized Dirac plasmons, $\omega_{ \pm}$or $\omega_{3}^{ \pm}$, giving rise to typical V-shaped wave patterns, containing some localized anomalies in the form of "holes" due to destructive interferences among the hybridized modes.

For large velocities $(v \gg 1)$, the charged particle only excites the even (symmetric) hybridized Dirac plasmons, $\omega_{+}$ or $\omega_{3}^{+}$. As a result, in this regime, the wake potential forms near-perfect, "single-harmonic" V-shaped wave patterns, lagging behind the charged particle. Besides slow oscillations in the wake potential (which come from the excitations of the Dirac plasmon in configurations with doped graphene or from excitations of the TO phonons in configurations with undoped graphene), there are fast oscillations in the wake potential, just behind the charged particle. Those oscillations were obtained in calculations using both the eHD and the ab initio methods. It was therefore proven that the fast oscillations, giving rise to the near-perfect, $\mathrm{V}$-shaped wave patterns in the wake potential just behind the charge particle, indeed represent the highenergy $\pi$ plasmon in graphene.

While our findings can be useful in the vibrational and valence EELS of layered heterostructures that include graphene by using the aloof electron beams within STEM, we have shown that various properties of the wake pattern can be used to gain theoretical insight into the plasmon-phonon hybridization in those structures by selectively choosing the relevant frequency range via the simple change of the incident particle velocity.

In order to keep the parameter space in the present work tractable, we have decided to hold several important parameters fixed at their respective typical values: the doping density (i.e., Fermi energy, $E_{F}$ ) of graphene, the thickness of the structure, $a$, and the distance of the charged particle, $z_{0}$. While a detailed study of the effects due to variations of all those parameters will be a topic of future work, we only note here that variations in $z_{0}$ are not expected to give rise to any substantial changes in our results on the wake potential, but can affect the magnitude of that potential. Specifically, one may surmise from the factor $\exp \left(-Q z_{0}\right)$ in Eq. (1) that increasing the value of $z_{0}$ would cause a suppression of contributions of large wave numbers to the wake potential. Accordingly, one expects that generally, increasing the distance $z_{0}$ would cause a reduction in the magnitude of the wake potential for the in-plane distances that are close to the projected position of the charged particle, as well as a reduction in the amplitude of oscillations behind the particle in the regime of low incident velocities, which is dominated by the phonon excitations.

\section{ACKNOWLEDGMENTS}

The authors thank I. Kupčić for useful discussions and invaluable advice. Computational resources were provided by the Donostia International Physics Center (DIPC) computing center. V.D. acknowledges support from the QuantiXLie Center of Excellence, a project cofinanced by the Croatian Government and European Union through the European Regional Development Fund - the Competitiveness and Cohesion Operational Programme (Grant No. KK.01.1.1.01.0004). 
This work is supported by the Ministry of Education, Science, and Technological Development of the Republic of Serbia (Grant No. 45005), the Serbia-Croatia bilateral project (Grant No. 337-00-205/2019-09/28), and the COST Action CA15107 (Grant No. 41392). L.K. also acknowledges support from the Ministry of Education, Science, and Technological Development of the Republic of Serbia (Grant No. 32039). Z.L.M. acknowledges support from the Natural Sciences and Engineering Research Council of Canada (Grant No. 201603689).
[1] V. N. Neelavathi, R. H. Ritchie, and W. Brandt, Phys. Rev. Lett. 33, 302 (1974).

[2] W. Brandt, A. Ratkowski, and R. H. Ritchie, Phys. Rev. Lett. 33, 1325 (1974).

[3] P. Sigmund, Particle Penetration and Radiation Effects, Vol. 2, Springer Series in Solid State Sciences, Vol. 179 (Springer, Heidelberg, 2014).

[4] Y.-N. Wang, H.-T. Qiu, and Z. L. Mišković, Phys. Rev. Lett. 85, 1448 (2000).

[5] S. Kawata, C. Deutsch, and Y. J. Gu, Phys. Rev. E 99, 011201(R) (2019).

[6] P. E. Batson and J. Bruley, Phys. Rev. Lett. 67, 350 (1991).

[7] F. J. Garcia de Abajo and P. M. Echenique, Phys. Rev. B 46, 2663 (1992).

[8] F. J. Garcia de Abajo and P. M. Echenique, Phys. Rev. B 48, 13399 (1993).

[9] Y. Susuki, H. Mukai, K. Kimura, and M. Mannami, Nucl. Instrum. Methods Phys. Res., Sect. B 48, 347 (1990).

[10] H. Winter, J. C. Poizat, and J. Remillieux, Nucl. Instrum. Methods Phys. Res., Sect. B 67, 345 (1992).

[11] T. Nandi, K. Haris, Hala, G. Singh, P. Kumar, R. Kumar, S. K. Saini, S. A. Khan, A. Jhingan, P. Verma, A. Tauheed, D. Mehta, and H. G. Berry, Phys. Rev. Lett. 110, 163203 (2013).

[12] G. Sharma, N. K. Puri, P. Kumar, and T. Nandi, Europhys. Lett. 120, 63002 (2017).

[13] A. G. Borisov, A. Mertens, H. Winter, and A. K. Kazansky, Phys. Rev. Lett. 83, 5378 (1999).

[14] A. A. Lucas, M. Šunjić, G. Benedek, and P. M. Echenique, New J. Phys. 16, 063015 (2014).

[15] Y.-N. Wang and T.-C. Ma, Phys. Rev. B 52, 16395 (1995).

[16] D. J. Mowbray, Z. L. Mišković, F. O. Goodman, and Y.-N. Wang, Phys. Lett. A 329, 94 (2004).

[17] I. Radović, D. Borka, and Z. L. Mišković, Phys. Lett. A 375, 3720 (2011).

[18] V. Despoja, K. Dekanić, M. Šunjić, and L. Marušić, Phys. Rev. B 86, 165419 (2012).

[19] I. Radović, D. Borka, and Z. L. Mišković, Phys. Rev. B 86, 125442 (2012).

[20] I. Radović, D. Borka, and Z. L. Mišković, Phys. Lett. A 377, 2614 (2013).

[21] X. Shi, X. Lin, F. Gao, H. Xu, Z. Yang, and B. Zhang, Phys. Rev. B 92, 081404(R) (2015).

[22] A. J. Chaves, N. M. R. Peres, G. Smirnov, and N. A. Mortensen, Phys. Rev. B 96, 195438 (2017).

[23] E. B. Kolomeisky and J. P. Straley, Phys. Rev. Lett. 120, 226801 (2018).

[24] I. Radović, V. Borka Jovanović, D. Borka, and Z. L. Mišković, Nucl. Instrum. Methods Phys. Res., Sect. B 279, 165 (2012).

[25] T. Marinković, I. Radović, D. Borka, and Z. L. Mišković, Plasmonics 10, 1741 (2015).
[26] T. Marinković, I. Radović, D. Borka, and Z. L. Mišković, Phys. Lett. A 379, 377 (2015).

[27] L.-J. Hou, Y.-N. Wang, and Z. L. Mišković, Phys. Rev. E 64, 046406 (2001).

[28] E. Takhtamirov and R. V. N. Melnik, Phys. Rev. B 84, 045313 (2011).

[29] P. Genevet, D. Wintz, A. Ambrosio, A. She, R. Blanchard, and F. Capasso, Nat. Nanotechnol. 10, 804 (2015).

[30] P. A. Crozier, Ultramicroscopy 180, 104 (2017).

[31] E. H. Hwang and S. Das Sarma, Phys. Rev. B 80, 205405 (2009).

[32] S. Das Sarma, S. Adam, E. H. Hwang, and E. Rossi, Rev. Mod. Phys. 83, 407 (2011).

[33] V. Despoja, D. Novko, K. Dekanić, M. Šunjić, and L. Marušić, Phys. Rev. B 87, 075447 (2013).

[34] D. Novko, M. Šunjić, and V. Despoja, Phys. Rev. B 93, 125413 (2016)

[35] M. Jablan, H. Buljan, and M. Soljačić, Phys. Rev. B 80, 245435 (2009).

[36] F. Bonaccorso, Z. Sun, T. Hasan, and A. C. Ferrari, Nat. Photonics 4, 611 (2010).

[37] A. Vakil and N. Engheta, Science 332, 1291 (2011).

[38] T. Low and P. Avouris, ACS Nano 8, 1086 (2014).

[39] H. Yan, X. Li, B. Chandra, G. Tulevski, Y. Wu, M. Freitag, W. Zhu, P. Avouris, and F. Xia, Nat. Nanotechnol. 7, 330 (2012).

[40] J. S. Gomez-Diaz, C. Moldovan, S. Capdevila, J. Romeu, L. S. Bernard, A. Magrez, A. M. Ionescu, and J. Perruisseau-Carrier, Nat. Commun. 6, 6334 (2015).

[41] Y. Francescato, V. Giannini, J. Yang, M. Huang, and S. A. Maier, ACS Photonics 1, 437 (2014).

[42] M. V. Fischetti, D. A. Neumayer, and E. A. Cartier, J. Appl. Phys. 90, 4587 (2001).

[43] H. Yan, T. Low, W. Zhu, Y. Wu, M. Freitag, X. Li, F. Guinea, P. Avouris, and F. Xia, Nat. Photonics 7, 394 (2013).

[44] Z. Fei, G. O. Andreev, W. Bao, L. M. Zhang, A. S. McLeod, C. Wang, M. K. Stewart, Z. Zhao, G. Dominguez, M. Thiemens, M. M. Fogler, M. J. Tauber, A. H. Castro-Neto, C. N. Lau, F. Keilmann, and D. N. Basov, Nano Lett. 11, 4701 (2011).

[45] L. Ju, B. Geng, J. Horng, C. Girit, M. Martin, Z. Hao, H. A. Bechtel, X. Liang, A. Zettl, Y. R. Shen, and F. Wang, Nat. Nanotechnol. 6, 630 (2011).

[46] N. Papasimakis, Z. Luo, Z. X. Shen, F. D. Angelis, E. D. Fabrizio, A. E. Nikolaenko, and N. I. Zheludev, Opt. Express 18, 8353 (2010).

[47] J. Niu, Y. J. Shin, J. Son, Y. Lee, J. H. Ahn, and H. Yang, Opt. Express 20, 19690 (2012).

[48] J. Niu, Y. J. Shin, Y. Lee, J. H. Ahn, and H. Yang, Appl. Phys. Lett. 100, 061116 (2012).

[49] T. Stauber, J. Phys.: Condens. Matter 26, 123201 (2014).

[50] V. Despoja, T. Djordjević, L. Karbunar, I. Radović, and Z. L. Mišković, Phys. Rev. B 96, 075433 (2017). 
[51] M. Liu, X. Yin, and X. Zhang, Nano Lett. 12, 1482 (2012).

[52] B. Xiao, R. Sun, Z. Xie, and X. Wang, Optoelectron. Adv. Mater., Rapid Commun. 9, 692 (2015).

[53] J. Burgdörfer, Nucl. Instrum. Methods Phys. Res., Sect. B 67, 1 (1992).

[54] B. Wunsch, T. Stauber, F. Sols, and F. Guinea, New J. Phys. 8, 318 (2006).

[55] E. H. Hwang and S. Das Sarma, Phys. Rev. B 75, 205418 (2007).

[56] K. F. Allison, D. Borka, I. Radović, Lj. Hadžievski, and Z. L. Mišković, Phys. Rev. B 80, 195405 (2009).

[57] D. J. Mowbray, S. Segui, J. Gervasoni, Z. L. Mišković, and N. R. Arista, Phys. Rev. B 82, 035405 (2010).

[58] T. Djordjević, I. Radović, V. Despoja, K. Lyon, D. Borka, and Z. L. Mišković, Ultramicroscopy 184, 134 (2018).

[59] A. Politano, I. Radović, D. Borka, Z. L. Mišković, and G. Chiarello, Carbon 96, 91 (2016).

[60] D. Novko, V. Despoja, and M. Šunjić, Phys. Rev. B 91, 195407 (2015).

[61] R. Nunez, P. M. Echenique, and R. H. Ritchie, J. Phys. C: Solid State Phys. 13, 4229 (1980).

[62] N. D. Mermin, Phys. Rev. B 1, 2362 (1970).

[63] Working in the Fourier domain, we combine the continuity equation in graphene, $\omega \rho=\mathbf{Q} \cdot \mathbf{j}$, Ohm's law for the induced current, $\mathbf{j}=\sigma(\omega) \mathbf{E}^{\text {tot }}$, the total electric field expressed as $\mathbf{E}^{\text {tot }}=-i \mathbf{Q} \phi^{\text {tot }}$, and the relation defining the response function, $\rho(Q, \omega)=\chi^{0}(Q, \omega) \phi^{\text {tot }}(Q, \omega)$, to obtain the expression $\chi^{0}(Q, \omega)=\frac{Q^{2}}{i \omega} \sigma(\omega)$.

[64] F. J. Nelson, J.-C. Idrobo, J. D. Fite, Z. L. Mišković, S. J. Pennycook, S. T. Pantelides, J. U. Lee, and A. C. Diebold, Nano Lett. 14, 3827 (2014).

[65] V. B. Jovanović, I. Radović, D. Borka, and Z. L. Mišković, Phys. Rev. B 84, 155416 (2011).

[66] P. Wachsmuth, R. Hambach, M. K. Kinyanjui, M. Guzzo, G. Benner, and U. Kaiser, Phys. Rev. B 88, 075433 (2013).

[67] J.-C. Idrobo and W. Zhou, Ultramicroscopy 180, 156 (2017).

[68] B. G. Frederick, G. Apai, and T. N. Rhodin, Phys. Rev. B 44, 1880 (1991).

[69] Z.-Y. Ong and M. V. Fischetti, Phys. Rev. B 86, 165422 (2012).

[70] R. Saito, G. Dresselhaus, and M. S. Dresselhaus, Physical Properties of Carbon Nanotubes (Imperial College Press, London, 1998).

[71] P. Giannozzi, S. Baroni, N. Bonini, M. Calandra, R. Car, C. Cavazzoni, D. Ceresoli, G. L. Chiarotti, M. Cococcioni, I. Dabo et al., J. Phys.: Condens. Matter 21, 395502 (2009).

[72] N. Troullier and J. L. Martins, Phys. Rev. B 43, 1993 (1991).

[73] J. P. Perdew and A. Zunger, Phys. Rev. B 23, 5048 (1981).

[74] H. J. Monkhorst and J. D. Pack, Phys. Rev. B 13, 5188 (1976).

[75] L. Marušić and V. Despoja, Phys. Rev. B 95, 201408(R) (2017).

[76] V. Despoja, Z. Rukelj, and L. Marušić, Phys. Rev. B 94, 165446 (2016).

[77] I. Lončarić, Z. Rukelj, V. M. Silkin, and V. Despoja, npj 2D Materials and Applications 2, 33 (2018). 